hep-th/0107140

IFT-P.052/2001

ITEP-TH-72/00

CITUSC/00-067

CALT-68-2312

\title{
Superstrings in 2D Backgrounds with R-R Flux and New Extremal Black Holes
}

\author{
Nathan Berkovits, 1 Sergei Gukov, 2012 and Brenno Carlini Vallilo 3 \\ - Instituto de Física Teórica, Universidade Estadual Paulista \\ Rua Pamplona 145, 01405-900, São Paulo, SP, Brasil \\ * Joseph Henry Laboratories, Princeton University, Princeton, NJ 08544 , \\ California Institute of Technology, Pasadena, CA 91125, USA
}

\begin{abstract}
The hybrid formalism is used to quantize the superstring compactified to twodimensional target-space in a manifestly spacetime supersymmetric manner. A quantizable sigma model action is then constructed for the Type II superstring in curved twodimensional supergravity backgrounds which can include Ramond-Ramond flux. Such curved backgrounds include Calabi-Yau four-fold compactifications with Ramond-Ramond flux, and new extremal black hole solutions in two-dimensional dilaton supergravity theory. These black hole solutions are a natural generalization of the CGHS model and might be possible to describe using a supergroup version of the $\mathrm{SL}(2, \mathrm{R}) / \mathrm{U}(1) \mathrm{WZW}$ model. We also study some dynamical aspects of the new black holes, such as formation and evaporation.
\end{abstract}

July 2001

\footnotetext{
1 E-Mail: nberkovi@ift.unesp.br

2 E-Mail: gukov@feynman.princeton.edu

3 E-Mail: vallilo@ift.unesp.br
} 


\section{Introduction}

Since Ramond-Ramond vertex operators in the Ramond-Neveu-Schwarz (RNS) formalism are represented by spin fields which mix the matter and ghost sector, it is difficult to use this formalism to quantize the superstring in Ramond-Ramond (R-R) backgrounds. Although the covariant Green-Schwarz (GS) formalism can classically describe R-R backgrounds, this formalism has quantization problems even in a flat background.

Over the last seven years, a hybrid formalism has been developed which combines advantages of the RNS and GS formalisms. Like the RNS formalism, the hybrid formalism has a free-field action in a flat background so it is easy to quantize. And like the GS formalism, the worldsheet variables include the target superspace coordinates $(x, \theta)$ so target-space supersymmetry is manifest and R-R backgrounds are easy to describe. Furthermore, the hybrid formalism is related by a field redefinition to the $N=1 \rightarrow N=2$ embedding of the RNS formalism [1] [2], and the resulting critical $\widehat{c}=2 N=2$ superconformal field theory (scft) splits into a $\widehat{c}=(d-6) / 2 N=2 \mathrm{scft}$ for the target space and a $\widehat{c}=(10-d) / 2 N=2 \mathrm{scft}$ for the internal compactification manifold. The $N=2 \mathrm{scft}$ for the internal compactification manifold is related to the analogous RNS $\widehat{c}=(10-d) / 2$ $N=2$ scft by twisting the $\mathrm{U}(1)$-charged compactification-dependent worldsheet fields. This hybrid formalism has been previously developed for compactification to $d=6$ on Calabi-Yau (CY) two-folds [2] [3] [4] and for compactification to $d=4$ on CY three-folds [5] [6]. It will be developed here for compactification to $d=2$ on CY four-folds.

Even though in two dimensions IIA R-R fields have no propagating degrees of freedom, their zero-modes play a very important role - they induce a back reaction on the graviton generating a negative cosmological constant in the effective two-dimensional theory. Therefore, in addition to describing CY four-fold compactifications with R-R flux, the hybrid formalism developed here can be used to study $D=2$ dilaton supergravity with an additional R-R term, which is a natural generalization of the CGHS model. This $D=2$ supergravity theory has new extremal black hole solutions which will be shown to have interesting properties.

The paper is organized as follows: In section 2, we develop the hybrid formalism for superstring compactification on CY four-folds to flat two-dimensional Minkowski space. For heterotic compactifications, $N=(2,0)$ spacetime supersymmetry is manifest, while for IIA (or IIB) compactifications, $N=(2,2)$ (or $N=(4,0)$ ) spacetime supersymmetry is manifest. After defining the free-field action and worldsheet $N=2$ superconformal 
generators in the hybrid formalism, vertex operators for physical massless states are explicitly constructed. For the Type II superstring, these massless states include four R-R field strengths which are independent of the compactification. These universal combinations of R-R fields will be identified with four possible types of F-terms in the effective $\mathcal{N}=2$ theory: superpotential, twisted superpotential, and their complex conjugates. As in [7], the hybrid formalism can be used to compute "topological" contributions to these superpotentials.

In section 3 , the superstring action and worldsheet $N=2$ superconformal generators are generalized for compactifications to a curved $d=2$ target-superspace background. The resulting sigma model action is manifestly target-space super-reparameterization invariant and can be used to quantize the superstring in two-dimensional backgrounds with R-R flux. The sigma model action contains a Fradkin-Tseytlin term which couples a spacetime superfield containing the dilaton to the worldsheet $N=(2,2)$ supercurvature. As in the $d=4$ and $d=6$ hybrid formalisms [6] [4], this coupling is possible since superspace chirality in spacetime is related to superspace chirality on the worldsheet. The background superfields appearing in the sigma model are then used to construct a low-energy superpace effective action for the $N=(2,2)$ supergravity theory.

In section 4 , we study the bosonic contribution to the low-energy effective action of the two-dimensional supergravity theory. A particular feature of this action is a (cosmological) constant term, independent of the dilaton, which is induced by the background R-R flux. The action without this term was extensively studied in the literature in the early 90s. In particular, it was shown that it has classical black hole solutions [8,9,10], which evaporate [11], and eventually become singular [12], in quantum theory coupled to matter. We show that with an extra term corresponding to the R-R flux, the theory admits a large family of novel black hole solutions including extremal black holes, analogous to those in [13]. We conjecture that the extremal solutions represent stable $1 / 2$ BPS states of the twodimensional supergravity theory and support this conjecture by calculating the mass of non-singular black hole solutions and demonstrating that it satisfies a BPS-like inequality. Then we examine some dynamical aspects of the new black holes, in the classical and semiclassical approximations. Namely, we study formation and evaporation of the nearextremal black holes and argue that the new extremal black holes do not emit Hawking radiation.

Finally, in section 5 we conclude with a discussion of open problems which include finding a supergroup sigma model to describe the superstring quantized on the black hole 
solutions of section 4. Such a sigma model could be useful for studying quantum corrections to the solutions, in a manner analogous to the $\mathrm{SL}(2, \mathrm{R}) / \mathrm{U}(1)$ gauged WZW sigma model description [10] of the CGHS black holes [9,11].

\section{Hybrid Formalism for Compactification to Two Flat Dimensions}

In this section we describe the basic methods of the hybrid formalism and how to apply them in the case of superstring compactification to a flat two-dimensional background. The first step will be to find a field redefinition from RNS variables to hybrid variables which allows $d=2$ spacetime supersymmetry to be made manifest. The next step will be to construct worldsheet $N=2$ superconformal generators in the hybrid formalism using the $N=1 \rightarrow N=2$ embedding of the RNS superstring. The final step will be to use these $N=2$ superconformal generators to define physical vertex operators in the hybrid formalism.

\subsection{Field redefinition from RNS variables}

The basic variables of the RNS description of the superstring are $\left(x^{m}, \psi^{m}\right)$ and the (bosonized) superconformal ghosts $\left(b, c, \beta=e^{-\phi} \partial \xi, \gamma=e^{\phi} \eta\right)$ which gauge-fix the $N=1$ supergravity of the worldsheet. For superstring compactifications which preserve at least $N=2$ supersymmetry in flat two-dimensional space-time, these variables factorize into a set describing the flat space $\left(x^{\ddagger}, x^{=}, \psi^{\ddagger}, \psi^{=}\right)$plus the superconformal ghosts and a $\widehat{c}=4$ $N=2$ scft associated with the eight-dimensional compactification manifold, e.g. a CalabiYau four-fold. The action has the form (for simplicity we will discuss only the left moving sector in this section):

$$
S_{\text {flat }}=\int d^{2} z\left[\partial x^{=} \bar{\partial} x^{\ddagger}-\psi^{\ddagger} \bar{\partial} \psi^{=}\right]+S_{C Y}+S_{\text {ghosts }},
$$

where $S_{\text {ghosts }}$ is the action for the superconformal ghosts and $S_{C Y}$ is the sigma model action for the compactification manifold, which we assume to be a Calabi-Yau four-fold for the sake of concreteness. The explicit form of these actions will not be needed.

As discussed in [14], spacetime supersymmetry can be studied defining the zero mode of the vertex operator of the massless fermionic state at zero momentum to be the supersymmetry charge. Since there are only two non-compact dimensions, the spin fields take a very simple form, $\Sigma^{\alpha}=e^{ \pm \frac{1}{2} \sigma}$ where $\sigma$ is the chiral boson which bosonize the $\left(\psi^{\ddagger}, \psi^{=}\right)$ 
variables. The GSO projection tells us that we must choose one chirality for $\Sigma^{\alpha}$ and we will choose $\Sigma^{-}$. In the $-\frac{1}{2}$ picture, there are two supercharges, $q_{+}=\oint e^{-\frac{1}{2} \phi} \Sigma^{-} e^{+\frac{1}{2} H_{C Y}}$ and $q_{\dot{+}}=\oint e^{-\frac{1}{2} \phi} \Sigma^{-} e^{-\frac{1}{2} H_{C Y}}$, where $J_{C Y}=\partial H_{C Y}$ is the worldsheet current contained in the $N=2$ superconformal algebra of the CY sigma model. The fact that the supersymmetry operator carries picture tell us that supersymmetry in the RNS model is well defined only for on-shell states. This can also be seen verifying that these operators satisfy the $d=2$ $N=2$ supersymmetry algebra up to a picture change.

Nevertheless, in two spacetime dimensions it is possible to define supersymmetry offshell using the methods of [5] [2] [3]. To do this, we define $q_{+}$in the $-\frac{1}{2}$ picture as above but define its complex conjugated $q_{+}$in the $\frac{1}{2}$ picture as

$$
q_{+}=\oint\left(b \eta e^{\frac{3}{2} \phi} \Sigma^{-} e^{\frac{1}{2} H_{C Y}}-e^{\frac{1}{2} \phi}:\left[\partial x^{=} \Sigma^{+}+\left(G_{C Y}^{+}+G_{C Y}^{-}\right) \Sigma^{-}\right] e^{\frac{1}{2} H_{C Y}}:\right),
$$

where $G_{C Y}^{+}$and $G_{C Y}^{-}$are superconformal generators of the CY sigma model. For this to be consistent, we must change the definition of complex conjugation. This can be done following [15], and will not be described explicitly here. The two generators $q_{\dot{+}}$ and $q_{+}$ satisfy the desired supersymmetry algebra.

Now we define two superspace variables $\theta^{+}$and $\theta^{+}$that when commuted with $q_{+}$and $q_{\dot{+}}$ respectively give the identity operator:

$$
\theta^{+}=c \xi e^{-\frac{3}{2} \phi} \Sigma^{+} e^{-\frac{1}{2} H_{C Y}}, \quad \theta^{\dot{+}}=e^{\frac{1}{2} \phi} \Sigma^{+} e^{\frac{1}{2} H_{C Y}} .
$$

Note that these operators have conformal weight zero and transform as spacetime spinors. In addition, we define conformal weight one operators conjugate to $\theta$ 's:

$$
p_{+}=b \eta e^{\frac{3}{2} \phi} \Sigma^{-} e^{\frac{1}{2} H_{C Y}}, \quad p_{\dot{+}}=e^{-\frac{1}{2} \phi} \Sigma^{-} e^{-\frac{1}{2} H_{C Y}} .
$$

We will treat these fields as the fundamental ones. Remembering the initial number of degrees of freedom, $\left(x^{\ddagger}, x^{=}, \psi^{\ddagger}, \psi^{=}, b, c, \beta, \gamma\right)$, we see that there are two independent chiral bosons remaining to be defined. These two chiral bosons, $\omega$ and $\rho$, will be defined as

$$
\omega=\frac{1}{2}(\phi-\kappa+\sigma-\chi), \quad \rho=2 \phi+\frac{1}{2}\left(H_{C Y}-\chi-3 \kappa\right),
$$

where $\partial \chi=c b$ and $\partial \kappa=\xi \eta$. These chiral bosons have the OPE's:

$$
\omega(z) \omega(w) \rightarrow \frac{1}{2} \log (z-w), \quad \rho(z) \rho(w) \rightarrow-\frac{1}{2} \log (z-w) .
$$


The set of hybrid variables is now complete. The next step is to make a chiral $U(1)$ rotation on the compactification fields in order they do not have singular OPE's with the hybrid variables due to the $H_{C Y}$ dependence. If a compactification field $\Phi_{C Y}$ has $U(1)$ charge $n$, we will define a new field $\Phi_{G S}=e^{n(\phi-\kappa)} \Phi_{C Y}$. This transformation changes the compactification dependent superconformal generators by a similarity transformation $\mathcal{O}_{G S}=e^{\oint(\phi-\kappa) J_{C Y}} \mathcal{O}_{C Y} e^{-\oint(\phi-\kappa) J_{C Y}}$. In particular, $G_{G S}^{ \pm}=e^{ \pm(\phi-\kappa)} G_{C Y}^{ \pm}$and $J_{G S}=$ $J_{C Y}+4(\partial \phi-\partial \kappa)$.

\subsection{Worldsheet $N=2$ superconformal generators}

As discussed in [1], the generators $\left(T=T_{\text {matter }}^{R N S}+T_{g h o s t}^{R N S}, G^{+}=j_{B R S T}, G^{-}=b, J=\right.$ $c b+\xi \eta)$ of the combined matter ghost system form an $N=2$ twisted superconformal algebra with central charge $\widehat{c}=2$. These generators can be untwisted by defining $T \rightarrow T-\frac{1}{2} \partial J$ and rewritten using the hybrid variables. Using the definition of $p_{+}, \omega$ and $\rho$, it is easy to see that $J=-2 \partial \rho+J_{G S}$ and $G^{-}=b=p_{+} e^{\omega-\rho}$.

$G^{+}=j_{B R S T}$ can be determined in a similar way (e.g. $-\gamma^{2} b=p_{\dot{+}} e^{\omega+\rho}$ ) and can be checked by requiring consistency of the algebra. $T$ is uniquely determined by conformal weights of the fundamental fields and requiring $G^{+}$and $G^{-}$to have conformal weight $\frac{3}{2}$. The complete set of untwisted generators is:

$$
\begin{gathered}
T=\Pi \neq \Pi=-d_{+} \partial \theta^{+}-d_{\dot{+}} \partial \theta^{\dot{+}}+\partial \omega \partial \omega-\partial \rho \partial \rho-\partial^{2} \omega+T_{G S}, \\
G^{-}=d_{+} e^{\omega-\rho}, \\
G^{+}=\left(\Pi_{=} d_{\dot{+}}+2 \partial \omega \partial \theta^{+}-\frac{1}{2} \partial^{2} \theta^{+}\right) e^{-\omega+\rho}+d_{\dot{+}} e^{\omega+\rho}+G_{G S}^{+}+\theta^{+} T_{G S} e^{-\omega+\rho}+G_{G S}^{-} \theta^{+} p_{\dot{+}} e^{2 \rho}, \\
J=-2 \partial \rho+J_{G S},
\end{gathered}
$$

where

$$
\begin{gathered}
\Pi_{=}=\partial x_{=}+\theta^{+} \partial \theta^{\dot{+}}, \quad \Pi_{\neq}=\partial x_{\neq}, \\
d_{+}=p_{+}, \quad d_{\dot{+}}=p_{\dot{+}}+\theta^{+} \partial x \neq,
\end{gathered}
$$

are supersymmetric operators.

This can be expressed in a more elegant form by doing a similarity transformation in the hybrid part

$$
\mathcal{O}_{\text {hybrid }} \rightarrow e^{\oint\left(\theta^{+} e^{\rho-\omega} G_{G S}^{-}-\frac{1}{2} \partial x \neq \theta^{+} \theta^{\dot{+}}\right)} \mathcal{O}_{\text {hybrid }} e^{-\oint\left(\theta^{+} e^{\rho-\omega} G_{G S}^{-}-\frac{1}{2} \partial x \ddagger \theta^{+} \theta^{\dot{+}}\right)} .
$$


Note that it does not change the conformal weight or the charge of the operators, puts a term $G_{G S}^{-}$in $G^{-}$and removes the terms $\theta^{+} T_{G S} e^{-\omega+\rho}+G_{G S}^{-} \theta^{+} p_{\dot{+}} e^{2 \rho}$ in $G^{+}$. This transformation also allows us to write the $d=2$ supersymmetric operators and supercharges as:

$$
\begin{gathered}
\Pi_{=}=\partial x_{=}+\frac{1}{2}\left(\theta^{+} \partial \theta^{\dot{+}}+\theta^{\dot{+}} \partial \theta^{+}\right), \quad \Pi_{\neq}=\partial x_{\ddagger}, \\
d_{+}=p_{+}+\frac{1}{2} \theta^{\dot{+}} \partial x_{\ddagger}, \quad d_{\dot{+}}=p_{\dot{+}}+\frac{1}{2} \theta^{+} \partial x \neq, \\
q_{+}=\int\left(p_{+}-\frac{1}{2} \theta^{\dot{+}} \partial x_{\ddagger}\right), \quad q_{\dot{+}}=\int\left(p_{\dot{+}}-\frac{1}{2} \theta^{+} \partial x_{\ddagger}\right)
\end{gathered}
$$

and the superconformal generators are

$$
\begin{gathered}
T=\Pi_{+} \Pi_{=}-d_{+} \partial \theta^{+}-d_{\dot{+}} \partial \theta^{\dot{+}}+\partial \omega \partial \omega-\partial^{2} \omega-\partial^{2} \rho+T_{G S}, \\
G^{-}=d_{+} e^{w-\rho}+G_{G S}^{-}, \\
G^{+}=e^{\oint \Pi=e^{-2 w}}\left(d_{\dot{+}} e^{\omega+\rho}\right) e^{-\oint \Pi=e^{-2 w}}+G_{G S}^{+}, \\
J=-2 \partial \rho+J_{G S},
\end{gathered}
$$

The worldsheet action for these hybrid variables is

$$
S_{h y b r i d}=\int d^{2} z\left[\partial x^{=} \bar{\partial} x^{\ddagger}-p_{+} \bar{\partial} \theta^{+}-p_{\dot{+}} \bar{\partial} \theta^{\dot{+}}\right]+S_{C}+S_{B}
$$

where $S_{C}$ is the action for the new $\widehat{c}=4 \mathrm{scft}$ and $S_{B}$ is the action for the chiral bosons $\rho$ and $w$. Like its RNS counterpart of (2.1), (2.12) is a free-field action if one ignores the scft for the compactification. But $S_{\text {hybrid }}$ has the advantage over (2.1) that the hybrid variables transform covariantly under the spacetime supersymmetry transformations generated by (2.10). In the closed string case there are also hybrid variables corresponding to the right movers. The difference between type IIA and IIB is the choice of the chiralities of the spinor variables. Note that the right mover chiral boson $\bar{\omega}$ has different $S O(1,1)$ charges in the two cases. 


\subsection{Definition of physical states}

With this superconformal algebra, one can introduce $N=2$ superconformal ghosts and carry out a standard BRST procedure to define physical states [5]. But since we have already included the RNS matter and ghost fields to define the hybrid variables, the $\mathrm{N}=2$ superconformal ghosts play a trivial role and will be ignored in the following discussion.

For open superstrings, physical vertex operators can be defined as real, chiral, or antichiral $N=2$ primary fields constructed from the hybrid variables. fields $\Phi$ carry zero $U(1)$ charge and zero conformal weight and satisfy $G_{n}^{+} \Phi=G_{n}^{-} \Phi=0$ for $n \geq 0$ where $\mathcal{O}_{n} \mathcal{A}$ means the coefficient of the pole of order $n+h_{\mathcal{O}}$ in the OPE of $\mathcal{O}$ and $\mathcal{A}$. Chiral primary fields $\Phi^{c}$ carry $+1 U(1)$ charge and $+\frac{1}{2}$ conformal weight and satisfy $G_{n-1}^{+} \Phi^{c}=G_{n}^{-} \Phi^{c}=0$ for $n \geq 0$, and antichiral primary fields $\Phi^{a}$ carry $-1 U(1)$ charge and $+\frac{1}{2}$ conformal weight and satisfy $G_{n}^{+} \Phi^{a}=G_{n-1}^{-} \Phi^{a}=0$ for $n \geq 0$. These primary fields are defined up to gauge transformations $\delta \Phi=G_{-n}^{+} \Lambda_{n}+G_{-n}^{-} \bar{\Lambda}_{n}$ for $n>0$ which preserve the above conditions.

For real primary fields, the integrated open superstring vertex operator is $V=$ $\int d z G_{-\frac{1}{2}}^{-} G_{-\frac{1}{2}}^{+} \Phi$, while for chiral and antichiral primary fields, the integrated open superstring vertex operator is $V=\int d z G_{-\frac{1}{2}}^{-} \Phi^{c}$ and $V=\int d z G_{-\frac{1}{2}}^{+} \Phi^{a}$. Physical closed superstring vertex operators can be defined in the usual manner as left-right products of open superstring vertex operators. For example, for real primary fields, the integrated closed superstring vertex operator is

$$
\mathcal{V}=\int d^{2} z \bar{G}_{-\frac{1}{2}}^{-} \bar{G}_{-\frac{1}{2}}^{+} G_{-\frac{1}{2}}^{-} G_{-\frac{1}{2}}^{+} \Phi
$$

\subsection{Compactification-independent massless states}

We will first discuss massless open string states independent of the compactification. The most general massless compactification-independent operator is

$$
\Phi(x, \theta)=V+U=e^{-2 w},
$$

4 An alternative way to define physical states in the hybrid formalism is to use the $N=4$ topological method [2] in which the $\widehat{c}=2 N=2$ generators are extended to a set of $\widehat{c}=2 N=4$ generators. Although this method is useful for constructing gauge-invariant actions or computing scattering amplitudes, it is unnecessary if one only wants to construct physical states. 
where $\Phi$ in the massless case depends only on the zero modes of $x$ and $\theta$, i.e. there are no derivatives on them. Using the real primary field conditions that $T_{0} \Phi=G_{\frac{1}{2}}^{ \pm} \Phi=0$ and the OPE's

$$
\begin{gathered}
x^{\ddagger}(y) x^{=}(z) \rightarrow 2 \log |y-z|, \quad w(y) w(z) \rightarrow \frac{1}{2} \log (y-z), \quad \rho(y) \rho(z) \rightarrow-\frac{1}{2} \log (y-z), \\
\theta^{\dot{+}}(y) p_{\dot{+}}(z) \rightarrow(y-z)^{-1}, \quad \theta^{+}(y) p_{+}(z) \rightarrow(y-z)^{-1},
\end{gathered}
$$

we get the following superfield equations:

$$
\begin{gathered}
\square V=\square U_{=}=0 \\
\nabla_{+} U_{=}=0, \\
\nabla_{\dot{+}}\left(U_{=}-\nabla_{=} V\right)=0,
\end{gathered}
$$

where

$$
\begin{gathered}
\nabla_{=}=\partial_{=}, \quad \nabla_{\ddagger}=\partial_{\ddagger}, \\
\nabla_{+}=\partial_{+}+\frac{1}{2} \theta^{\dot{+}} \partial_{\ddagger}, \quad \nabla_{\dot{+}}=\partial_{\dot{+}}+\frac{1}{2} \theta^{+} \partial_{\neq} .
\end{gathered}
$$

The residual gauge invariance from $\delta \Phi=G_{-\frac{1}{2}}^{+} \Lambda+G_{-\frac{1}{2}}^{-} \bar{\Lambda}$ are generated by $\Lambda=$ $e^{-w-\rho} \Lambda_{-}, \quad \bar{\Lambda}=e^{-w+3 \rho-H_{C Y}} \Lambda_{-}$, with the gauge transformations:

$$
\begin{gathered}
\delta V=\nabla_{+} \Lambda_{-}+\nabla_{\dot{+}} \Lambda_{\dot{-}}, \\
\delta U_{=}=\nabla_{=} \nabla_{\dot{+}} \Lambda_{\dot{-}},
\end{gathered}
$$

satisfying $\nabla_{=} \nabla_{+} \nabla_{\dot{+}} \Lambda_{\dot{-}}=\nabla_{=} \nabla_{\dot{+}} \nabla_{+} \Lambda_{-}=0$.

The solution of the equations is

$$
\Phi=\theta^{+} \phi^{-}\left(x^{=}\right)+\theta^{\dot{+}} \phi^{\dot{-}}\left(x^{=}\right)+\theta^{\dot{+}} \partial_{=} \phi^{\dot{-}}\left(x^{=}\right) e^{-2 \omega} .
$$

The fields that are not eliminated by the equations of motion are pure gauge. Only the Ramond sector contains physical degrees of freedom which are two left-moving fermions, agreeing with the standard light-cone analysis.

In the case of closed Type II superstrings, the situation is unusual. Since the states of the closed string are just the product of the open string states, the physical degrees of freedom are in the R-R sector. For Type IIB we will get four chiral bosons (depending on $x^{=}$), but for Type IIA we will have only constant modes since the product of two chiral fermions of opposite chiralities ( the left moving contribution will depend on $x^{=}$and the right moving will depend on $x^{\ddagger}$ ) must be constant. Since constant R-R fluxes play an important role in $\mathrm{CY}$ four-fold compactifications, it will be interesting to discuss these compactification-independent states in more detail. 


\subsection{Type IIA $R$ - $R$ vertex operators}

When $\partial_{=} \phi^{-}$and $\partial=\phi^{-}$are constants in (2.17), the integrated vertex operators $V=\int d z G_{-\frac{1}{2}}^{-} G_{-\frac{1}{2}}^{+} \Phi$ associated with these open superstring states are the spacetime supersymmetry generators $q_{+}$and $q_{+}$of (2.10). The Type IIA vertex operators for the four constant R-R field-strengths are therefore

$$
V_{c c}=\int d^{2} z j_{+} j_{-}, \quad V_{a a}=\int d^{2} z j_{\dot{+}} j_{\dot{-}}, \quad V_{c a}=\int d^{2} z j_{+} j_{\dot{-}}, \quad V_{a c}=\int d^{2} z j_{\dot{+}} j_{-},
$$

where $q_{ \pm}=\oint j_{ \pm}$and $q_{ \pm}=\oint j_{ \pm}$are the four Type IIA supercharges.

In terms of the original 32 ten-dimensional IIA spacetime-supersymmetry generators $q_{\alpha}=q_{ \pm \pm \pm \pm \pm}$,

$$
q_{+}=q_{+++++}, \quad q_{\dot{+}}=q_{+----}, \quad q_{-}=q_{-++++}, \quad q_{\dot{-}}=q_{-----} .
$$

The vertex operator described by $\int d^{2} z j_{\alpha} j_{\beta}$ couples to the string coupling constant $e^{\varphi}$ times the ten-dimensional IIA R-R field strengths in the combination

$$
F_{\alpha \beta}=\delta_{\alpha \beta} F^{(0)}+\frac{1}{2} \gamma_{\alpha \beta}^{M N} F_{M N}^{(2)}+\frac{1}{24} \gamma_{\alpha \beta}^{M N P Q} F_{M N P Q}^{(4)}
$$

where $M=0$ to 9 . Since they carry +4 and -4 CY $U(1)$ charge, the compactificationindependent R-R vertex operators $V_{c c}$ and $V_{a a}$ are easily seen to couple to the holomorphic and anti-holomorphic four-form R-R field strengths $\Omega^{j k l m} F_{j k l m}^{(4)}$ and $\Omega^{\overline{j k l} \bar{m}} F_{\overline{j k l} \bar{m}}^{(4)}$ where $j, k, l, m=1$ to 4 denote the complex Calabi-Yau directions, and $\Omega$ is the covariantly constant $(4,0)$-form. In other words, $V_{c c}$ and $V_{a a}$ couple to the following components of the Ramond-Ramond field strengths:

$$
F_{+-}=W, \quad F_{\dot{+}}=\bar{W}
$$

where, following the notations of [16], we introduced:

$$
W=\frac{1}{2 \pi} \int_{X} \Omega \wedge F^{(4)}
$$

Similarly, one can check that the two other compactification-independent R-R vertex operators, $V_{c a}$ and $V_{a c}$, couple to $e^{\varphi}$ times two specific linear combinations of the field strengths $F^{(0)}, F_{01}^{(2)}, F_{j \bar{j}}^{(2)}, F_{01 j \bar{j}}^{(4)}$ and $F_{j k \overline{j k}}^{(4)}$. The precise linear combination of field strengths which couple to $V_{c a}$ and $V_{a c}$ can be derived using the mirror map of the CY 
manifold which maps the holomorphic $F_{j k l m}^{(4)}$ and the anti-holomorphic $F_{\overline{j k l} \bar{m}}^{(4)}$ into these linear combinations. The idea is that the conformal field theory associated with the mirror variety $\tilde{X}$ is physically equivalent to the CFT associated with the original CY four-fold $X$. Essentially, the two are related by a change of notations [17, 18], which exchanges $-\leftrightarrow \dot{-}$. This transformation implies a non-trivial isomorphism of the cohomology rings of $X$ and its mirror $\tilde{X}$, such that:

$$
h^{p, q}(X)=h^{4-p, q}(\widetilde{X})
$$

Therefore, we claim that R-R flux corresponding, say, to $V_{c a}$ is given by the image of $W$ on $\widetilde{X}$ under the mirror map, which we now describe in more detail.

Mirror symmetry is usually stated as a map from the moduli space $\mathcal{M}_{c}(X)$ of complex structure of $X$ to the moduli space of Kähler structure $\mathcal{M}_{K}(\widetilde{X})$ of the mirror manifold $\widetilde{X}$ [19]. Following [20], we denote by $D$ a connection on the holomorphic bundle over the complex structure moduli space $\mathcal{M}_{c}(X)$. Starting with the holomorphic 4-form $\Omega$, we can act on it with $D$ to generate the cohomology elements of $\oplus_{k=0}^{4} H^{k, 4-k}(X)$. The space spanned by these elements is called the "horizontal primary subspace":

$$
H_{H}(X) \subset \oplus_{k=0}^{4} H^{k, 4-k}(X) .
$$

Similarly, one can consider wedge products of the elements $\mathcal{O}_{i} \in H^{1,1}(\widetilde{X})$ in the cohomology of the mirror variety $\tilde{X}$. The subspace of $\oplus_{k=0}^{4} H^{k, k}(\tilde{X})$ spanned by these elements is called the "vertical primary subspace":

$$
H_{V}(\widetilde{X}) \subset \oplus_{k=0}^{4} H^{k, k}(\widetilde{X})
$$

Mirror symmetry for Calabi-Yau four-folds states that these two spaces we have just introduced, $H_{H}(X)$ and $H_{V}(\tilde{X})$, are isomorphic. We denote this isomorphism as:

$$
\pi_{m}: H_{H}(\tilde{X}) \longrightarrow H_{V}(X)
$$

Since the RR vertex operators $V_{c a}$ and $V_{a c}$ are related to $V_{c c}$ and $V_{a a}$ by mirror map in the world-sheet conformal field theory, the corresponding Ramond-Ramond fields must be also related by the mirror map (2.24). Therefore, the RR vertex operators $V_{c a}$ and $V_{a c}$ represent the following compactification-independent components of the Ramond-Ramond fields:

$$
\begin{aligned}
& F_{+\dot{-}}=\widetilde{W}_{X}=\pi_{m}\left(W_{\widetilde{X}}\right), \\
& F_{\dot{+}-}=\widetilde{W}_{X}=\pi_{m}\left(\overline{W_{\widetilde{X}}}\right)
\end{aligned}
$$

Using the mirror map (2.24) we can explicitly write down the expression for $\widetilde{W}$ 21]:

$$
\widetilde{W}=\frac{1}{2 \pi} \int_{X} e^{\mathcal{K}} \wedge\left(F^{(0)}+i F^{(2)}+F^{(4)}+i F^{(6)}+F^{(8)}\right)
$$

where $\mathcal{K}$ is the Kähler form on the CY space $X, F^{(6)}=* F^{(4)}$ and $F^{(8)}=* F^{(2)}$. 


\subsection{Compactification-dependent massless states}

For open string compactification dependent states, the massless states are given by chiral and antichiral primary vertex operators described by $\Phi^{c}=\left(V_{i}+e^{-2 w} U_{=i}\right) \Psi_{c}^{i}$ and $\Phi^{a}=\left(\bar{V}_{\bar{i}}+e^{-2 w} \bar{U}_{=\bar{i}}\right) \Psi_{a}^{\bar{i}}$ operators where $\Psi_{c}^{i}$ and $\bar{\Psi}_{a}^{\bar{i}}$ are chiral and antichiral left-moving primary operators of the CY scft. The chiral and antichiral primary conditions, $G_{\frac{1}{2}}^{-} \Phi^{c}=$ $G_{-\frac{1}{2}}^{+} \Phi^{c}=0$ and $G_{\frac{1}{2}}^{+} \Phi^{a}=G_{-\frac{1}{2}}^{-} \Phi^{a}=0$, imply the superfield equations

$$
\begin{gathered}
U_{=i}=-\nabla_{=} V_{i}, \quad \nabla_{+} V_{i}=0, \quad \nabla_{=} \nabla_{\dot{+}} V_{i}=0, \\
\bar{U}_{=\bar{i}}=0, \quad \nabla_{=} \nabla_{+} \bar{V}_{\bar{i}}=0, \quad \nabla_{\dot{+}} \bar{V}_{\bar{i}}=0 .
\end{gathered}
$$

The solution of these equations is:

$$
\begin{aligned}
& V_{i}=a\left(x^{=}, x^{\ddagger}\right)+\theta^{\dot{+}} b\left(x^{\ddagger}\right)-\frac{1}{2} \theta^{+} \theta^{\dot{+}} \partial_{\ddagger} a\left(x^{=}, x^{\ddagger}\right) \\
& \bar{V}_{\bar{i}}=\bar{a}\left(x^{=}, x^{\ddagger}\right)+\theta^{+} \bar{b}\left(x^{\ddagger}\right)+\frac{1}{2} \theta^{+} \theta^{\dot{+}} \partial_{\ddagger} \bar{a}\left(x^{=}, x^{\ddagger}\right)
\end{aligned}
$$

which is an $\mathrm{N}=(2,0) d=2$ scalar multiplet containing two real bosons and two left-moving fermions.

The closed string compactification-dependent states are obtained by taking the leftright product of open string states. For each $(c, c)$ or $(c, a) \mathrm{CY}$ modulus, the Type IIB superstring has a massless $\mathrm{N}=(4,0) d=2$ multiplet containing two real bosons, four leftmoving fermions, and two left-moving R-R bosons. For each $(c, c)$ or $(c, a)$ CY modulus, the Type IIA superstring has a massless $\mathrm{N}=(2,2) d=2$ scalar multiplet containing two real bosons, two left-moving fermions, two right-moving fermions, and two constant R-R bosons. As will be discussed in the following subsection, the R-R bosons in these multiplets play an interesting role in the low-energy superpotential terms.

\subsection{Computation of superpotential terms}

In the large volume limit, one can perform Kaluza-Klein reduction of type IIA supergravity on a Calabi-Yau space $X$ with RR flux in order to find the effective action for $2 D$ bosonic fields. The bosonic part of the effective Lagrangian of massive Type IIA supergravity in string frame looks as follows [22,23]:

$$
L_{I I A}=\int d^{10} x \sqrt{-g}\left[e^{-2 \varphi}\left(R+4(\nabla \varphi)^{2}-\frac{1}{12} H^{2}\right)-\sum_{p} \frac{1}{2 \cdot(2 p) !}\left(F^{(2 p)}\right)^{2}+\ldots\right]
$$


Here the dots stand for higher derivative $R^{4}$ and other terms. Even though we don't write these terms explicitly, they play an important role in the anomaly cancellation condition. Taking into account the back reaction effects of the RR flux, such as warping of the spacetime geometry, from the dimensional reduction of (2.27) one can obtain the bosonic part of the effective $\mathcal{N}=(2,2)$ two-dimensional dilaton supergravity interacting with matter 24]. In particular, one finds the effective superpotential (2.22) induced by the RR flux (along with the twisted chiral superpotential (2.26) ) [16,25,26]:

$$
W=\frac{1}{2 \pi} \int_{X} \Omega \wedge F^{(4)}
$$

This expression for the effective superpotential is protected by supersymmetry and can be also derived from (topological) string amplitudes. The manifestly supersymmetric hybrid approach turns out to be particularly useful for this sort of calculations [2]. Here we illustrate it for the case when $F^{(4)}$-flux is a linear combination of $(3,1)$ and $(1,3)$ forms, cf. [7]. The space of $(3,1)$ forms, $H^{3,1}(X)$, is generated by $\omega_{i}^{(3,1)}=D_{\phi_{i}} \Omega$ where $\left\{\phi_{i}\right\}$ are special coordinates on the complex structure moduli space $\mathcal{M}_{c}(X)$. Therefore, we can take R-R flux in the following form:

$$
F^{(3,1)}=\sum_{i} n_{i}\left(D_{\phi_{i}} \Omega\right)
$$

Recall, that for a generic CY four-fold compactification of type IIA superstring, complex structure moduli give rise to chiral superspace multiplets in the two-dimensional effective field theory (i.e. massless $\mathcal{N}=(2,2)$ superfields $\Phi_{i}\left(x^{\mu}, \theta^{+}, \theta^{-}\right)$satisfying $\left.\nabla_{\dot{+}} \Phi_{i}=\nabla_{\dot{-}} \Phi=0\right)$. From the Kaluza-Klein reduction one finds the superspace action of these modes [24]:

$$
S^{(2)}=\int d^{2} x d^{4} \theta e^{-K\left(\Phi_{i}, \bar{\Phi}_{i}\right)}=\int d^{2} x d^{4} \theta\left(\int_{X} \Omega \wedge \bar{\Omega}\right)
$$

where we ignored interaction with gravity. From the supersymmetry structure of the vertex operators for the fields $\Phi_{i}$ it follows that the auxiliary field in $\Phi_{i}$ corresponds to a Ramond-Ramond flux, so that we have:

$$
\Phi_{i}=\phi_{i}+(\theta)^{2} n_{i}+\ldots
$$

5 This notation means that $F^{(m, n)}$ contains $m$ holomorphic and $n$ anti-holomorphic indices in the $\mathrm{CY}$ directions. 
Turning on a R-R flux of the form (2.29) means giving a vev to the auxiliary fields in $\Phi_{i}$. Therefore, apart from the usual kinetic action for $\phi_{i}$, integrating over two $\theta$ variables in (2.30) we also get an F-term:

$$
\int d^{2} x d^{2} \theta\left(\sum_{i} n_{i} \int_{X} D_{\phi_{i}} \Omega \wedge \bar{\Omega}\right)+c . c .
$$

As in [7], this expression is exactly the same as the F-term that one finds after substituting (2.29) into superpotential (2.28). In a similar way, one should be able to reproduce other relations between D-terms and F-terms in the two-dimensonal effective action from (topological) string amplitudes controlled by supersymmetry $\mathbf{6}$.

\section{Hybrid Formalism in Curved $d=2$ Target-Space Background}

The usual method for constructing the superstring action in a curved target-space background is to add the on-shell massless vertex operator to the action in a flat background, and covariantize with respect to target-space (super-)reparameterizations. This procedure is not very useful for two-dimensional target-space backgrounds where almost all massless vertex operators are trivial on-shell. Nevertheless, it is easy to guess the correct superstring action in a curved two-dimensional target-superspace by simply changing the range of the superspace indices in the hybrid actions for the superstring in a curved four-dimensional [6] or six-dimensional [4] background.

As in the four and six-dimensional cases, the sigma model action in a two-dimensional background splits into a "classical" term and a Fradkin-Tseytlin term which couples the spacetime dilaton to the worldsheet $N=(2,2)$ supercurvature. After describing these two terms, it will be argued that worldsheet $N=(2,2)$ superconformal invariance of this action implies the appropriate low-energy superspace equations of motion for the background superfields.

6 An example of such relations is a generalization of the special geometry constraints, which by analogy with [27,28] can be formulated as a set of equations for the metric $G_{i \bar{j}}$ on the complex structure moduli space of $X$ and holomorphic four-point correlation function $\kappa_{i j k l}$ [20]:

$$
R_{i \bar{j} k \bar{l}}-G_{i \bar{j}} G_{k \bar{l}}-G_{i \bar{l}} G_{k \bar{j}}=e^{K} B^{p q \bar{s} \bar{t}} \kappa_{p q i k} \bar{\kappa}_{\bar{s} \bar{j} \bar{l}}
$$

Here $R_{i \bar{j} k \bar{l}}=-\bar{\partial}_{\bar{j}}\left(G_{k \bar{m}} \partial_{i} G^{\bar{m} n}\right) G_{n \bar{l}}$ is the curvature, and $\left(B^{-1}\right)_{p q \bar{s} \bar{t}}=e^{-K}\left(G_{p \bar{t}} G_{q \bar{s}}+G_{q \bar{t}} G_{p \bar{s}}-\right.$ $\left.R_{p \bar{t} q \bar{s}}\right)$, provided that $h_{\text {prim }}^{2,2}=h^{3,1}\left(h^{3,1}+1\right) / 2$. In type IIA compactifications on Calabi-Yau four-folds the use of such relations was first pointed out in [29]. 


\subsection{Classical sigma model action}

For the Type IIA superstring compactified on a Calabi-Yau four-fold, the "classical" part of the worldsheet action is

$$
\begin{gathered}
S=\frac{1}{\alpha^{\prime}} \int d z d \bar{z}\left(\Pi_{\mp} \bar{\Pi}_{=}+B_{A B} \Pi^{A} \bar{\Pi}^{B}\right. \\
+d_{+} \bar{\Pi}^{+}+\bar{d}_{\dot{+}} \bar{\Pi}^{\dot{+}}+d_{-} \Pi^{-}+\bar{d}_{\dot{-}} \Pi^{\dot{-}} \\
\left.+d_{+} d_{-} Y_{c c}+\bar{d}_{\dot{+}} \bar{d}_{\dot{-}} Y_{a a}+d_{+} \bar{d}_{\dot{-}} Y_{c a}+\bar{d}_{\dot{+}} d_{-} Y_{a c}\right) \\
+S_{C}+S_{B}
\end{gathered}
$$

where $\Pi^{A}=E_{M}^{A} \partial Z^{M}, \bar{\Pi}^{A}=E_{M}^{A} \bar{\partial} Z^{M}, A, B$ range over the tangent superspace indices $(\neq,=,+, \dot{+},-, \dot{-}), M$ ranges over the curved superspace indices, $Z^{M}=(x, \theta, \bar{\theta})$ are the $d=2 N=(2,2)$ target superspace coordinates, and $S_{C}$ and $S_{B}$ are actions for the compactification manifold and for the chiral bosons. Worldsheet fields carrying - or spinor indices come from the right-moving sector of the IIA superstring and those carrying + or $\dot{+}$ spinor indices come from the left-moving sector. Since the worldsheet variables $p_{ \pm}$and $\bar{p}_{ \pm}$never appear explicitly in (3.1), $d_{ \pm}$and $\bar{d}_{ \pm}$can be treated as independent worldsheet variables. Note that the background superfields $\left[Y_{c c}, Y_{a a}, Y_{c a}, Y_{a c}\right]$ need to be included in (3.1) to reproduce the R-R vertex operators of (2.18).

Although $S_{C}$ is the same action as in a flat $d=2$ background, $S_{B}$ needs to be modified since the chiral and antichiral bosons $(\omega, \rho, \bar{\omega}, \bar{\rho})$ are no longer free worldsheet fields. Since they carry Lorentz weight and $R$-charge, they couple to the $S O(1,1)$ Lorentz and $U(1) \times U(1)$ connections of the target-space supergravity theory which will be called $A_{M}^{L}, A_{M}^{1}$ and $A_{M}^{2}$. From their Lorentz and $R$-charges, one finds that the action $S_{B}$ in a curved background is

$$
S_{B}=S_{B}^{f l a t}+\int d z d \bar{z}\left[\bar{\partial} Z^{M}\left(\partial \omega A_{M}^{L}+\partial \rho A_{M}^{1}\right)+\partial Z^{M}\left(-\bar{\partial} \bar{\omega} A_{M}^{L}+\bar{\partial} \bar{\rho} A_{M}^{2}\right)\right]
$$

where $S_{B}^{\text {flat }}$ is the action for free chiral and antichiral bosons.

The background superfields appearing in (3.1) and (3.2) are the $d=2 N=$ $(2,2)$ target-space super-vierbein $E_{M}^{A}(Z)$, the Lorentz and $U(1) \times U(1)$ connections

7 For convenience, we will only discuss here the sigma model action for the Type IIA superstring. However, it is easy to generalize this action to the heterotic or Type IIB superstrings as was discussed in [6]. 
$\left[A_{M}^{L}(Z), A_{M}^{1}(Z), A_{M}^{2}(Z)\right]$, the tensor two-form superfield $B_{A B}(Z)$, and the four scalar superfields $\left[Y_{c c}(Z), Y_{a a}(Z), Y_{c a}(Z), Y_{a c}(Z)\right]$ whose lowest components are the string coupling constant times the four compactification-independent R-R field strengths discussed in subsection 2.5. As will be discussed in subsection 3.4, worldsheet $N=(2,2)$ superconformal invariance of the sigma model is expected to relate these background superfields to each other and impose their torsion constraints and low-energy equations of motion.

Note that (3.1) is manifestly target-space super-reparameterization invariant and reduces to the free-field action of (2.12) when the background superfields take their flat values. Although (3.1) has been written in worldsheet conformal gauge, it is easy to write it in a manifestly worldsheet reparameterization invariant manner by coupling appropriately to the worldsheet vierbein. However, since the worldsheet variables do not transform linearly under worldsheet $N=(2,2)$ supersymmetry (i.e. under commutation with $G_{-\frac{1}{2}}^{ \pm}$and $\bar{G}_{-\frac{1}{2}}^{ \pm}$), it is not known how to write (3.1) in a manifestly worldsheet superreparameterization invariant manner.

\subsection{Fradkin-Tseytlin term}

Because the expectation value of the spacetime dilaton is the logarithm of the string coupling constant, one expects a Fradkin-Tseytlin term which couples this spacetime field to the worldsheet curvature. As in the four and six-dimensional hybrid formalisms, this term is constructed by coupling chiral and twisted-chiral spacetime superfields containing the dilaton to chiral and twisted-chiral worldsheet superfields which describe the worldsheet $N=(2,2)$ supercurvature. This will be done in a consistent manner since worldsheet superspace chirality is related to target-superspace chirality in the hybrid formalism. So the Fradkin-Tseytlin term couples the target-space and worldsheet $N=2 d=2$ supergravities to each other.

In the $U(1) \times U(1)$ version of $N=2$ supergravity, the worldsheet supercurvature is described by a chiral and twisted-chiral superfield, $\Sigma_{c c}$ and $\Sigma_{c a}$, and their complex conjugates $\Sigma_{a a}$ and $\Sigma_{a c}$, satisfying

$$
\begin{aligned}
& D_{\dot{+}} \Sigma_{c c}=D_{\dot{-}} \Sigma_{c c}=0, \quad D_{\dot{+}} \Sigma_{c a}=D_{-} \Sigma_{c a}=0, \\
& D_{+} \Sigma_{a a}=D_{-} \Sigma_{a a}=0, \quad D_{+} \Sigma_{a c}=D_{-} \Sigma_{a c}=0 .
\end{aligned}
$$


$D_{ \pm}$and $D_{ \pm}$are the worldsheet $N=(2,2)$ supersymmetric derivatives which satisfy $\left\{D_{+}, D_{\dot{+}}\right\}=\partial_{z}$ and $\left\{D_{-}, D_{\dot{-}}\right\}=\bar{\partial}_{\bar{z}}$ on a flat worldsheet. The ordinary worldsheet curvature is given by the lowest component of $\operatorname{Re}\left(D_{+} D_{-} \Sigma_{c c}+D_{+} D_{-} \Sigma_{c a}\right)$, and the two worldsheet $U(1)$ field strengths are given by the lowest component of $\operatorname{Im}\left(D_{+} D_{-} \Sigma_{c c}+D_{+} D_{-} \Sigma_{c a}\right)$ and $\operatorname{Im}\left(D_{+} D_{-} \Sigma_{c c}+D_{\dot{+}} D_{-} \Sigma_{a c}\right)$.

The Fradkin-Tseytlin term is constructed by coupling these worldsheet superfields to the chiral and twisted-chiral spacetime superfields, $\Phi_{c c}$ and $\Phi_{c a}$, and their complex conjugates $\Phi_{a a}$ and $\Phi_{a c}$. These spacetime superfields satisfy

$$
\begin{aligned}
& \nabla_{\dot{+}} \Phi_{c c}=\nabla_{\dot{-}} \Phi_{c c}=0, \quad \nabla_{\dot{+}} \Phi_{c a}=\nabla_{-} \Phi_{c a}=0, \\
& \nabla_{+} \Phi_{a a}=\nabla_{-} \Phi_{a a}=0, \quad \nabla_{+} \Phi_{a c}=\nabla_{-} \Phi_{a c}=0
\end{aligned}
$$

where $\nabla_{ \pm}$and $\nabla_{ \pm}$are the spacetime $N=(2,2)$ supersymmetric derivatives satisfying $\left\{\nabla_{+}, \nabla_{\dot{+}}\right\}=\nabla_{\ddagger}$ and $\left\{\nabla_{-}, \nabla_{\dot{-}}\right\}=\nabla_{=}$. The lowest component of $\operatorname{Re}\left(\Phi_{c c}+\Phi_{c a}\right)$ is the spacetime dilaton, while the lowest component of $\operatorname{Im}\left(\Phi_{c c}+\Phi_{c a}\right)$ and $\operatorname{Im}\left(\Phi_{c c}+\Phi_{a c}\right)$ are spacetime $U(1) \times U(1)$ compensators which will be discussed in subsection 3.4.

As in the four and six-dimensional versions of the hybrid formalism, the FradkinTseytlin term is defined as

$$
\begin{aligned}
S_{F T} & =\int d^{2} z\left[G_{-\frac{1}{2}}^{-} \bar{G}_{-\frac{1}{2}}^{-}\left(\widehat{\Phi}_{c c} \Sigma_{c c}\right)+G_{-\frac{1}{2}}^{-} \bar{G}_{-\frac{1}{2}}^{+}\left(\widehat{\Phi}_{c a} \Sigma_{c a}\right)\right. \\
& \left.+G_{-\frac{1}{2}}^{+} \bar{G}_{-\frac{1}{2}}^{+}\left(\widehat{\Phi}_{a a} \Sigma_{a a}\right)+G_{-\frac{1}{2}}^{+} \bar{G}_{-\frac{1}{2}}^{-}\left(\widehat{\Phi}_{a c} \Sigma_{a c}\right)\right]
\end{aligned}
$$

where $G_{-\frac{1}{2}}^{ \pm} \widehat{\Phi}$ or $\bar{G}_{-\frac{1}{2}}^{ \pm} \widehat{\Phi}$ is defined using the OPE's of the worldsheet fields and $G_{-\frac{1}{2}}^{ \pm} \Sigma$ or $\bar{G}_{-\frac{1}{2}}^{ \pm} \Sigma$ is defined as the appropriate worldsheet supersymmetric derivative acting on $\Sigma$. In other words, treating the fermionic $N=2$ superconformal generators as worldsheet supersymmetry derivatives, one defines

$$
G_{-\frac{1}{2}}^{+} \Sigma=D_{+} \Sigma, \quad G_{-\frac{1}{2}}^{-} \Sigma=D_{\dot{+}} \Sigma, \quad \bar{G}_{-\frac{1}{2}}^{+} \Sigma=D_{-} \Sigma, \quad \bar{G}_{-\frac{1}{2}}^{-} \Sigma=D_{\dot{-}} \Sigma,
$$

since $+/-$ and $\dot{+} / \dot{-}$ target-space and worldsheet spinor indices correspond to unbarred/barred (or left/right moving) superconformal generators. The hatted $\Phi$ superfield is related to the unhatted $\Phi$ superfield by

$$
\widehat{\Phi}_{c c}=\left(1+e^{-2 \omega} \nabla_{\ddagger}+e^{-2 \bar{\omega}} \nabla_{=}+e^{-2 \omega-2 \bar{\omega}} \nabla_{\ddagger} \nabla_{=}\right) \Phi_{c c}, \quad \widehat{\Phi}_{a a}=\Phi_{a a}
$$




$$
\widehat{\Phi}_{c a}=\left(1+e^{-2 \omega} \nabla_{\ddagger}\right) \Phi_{c a}, \quad \widehat{\Phi}_{a c}=\left(1+e^{-2 \bar{\omega}} \nabla_{=}\right) \Phi_{a c} .
$$

The $e^{-\omega}$ factors are included in (3.7) so that (3.4) implies that $\widehat{\Phi}_{c c}$ satisfies $G_{-\frac{1}{2}}^{+} \widehat{\Phi}_{c}=$ $\bar{G}_{-\frac{1}{2}}^{+} \widehat{\Phi}_{c}=0$ and is therefore a chiral worldsheet field. Similarly, (3.4) implies that $\widehat{\Phi}_{c a}$ is a twisted-chiral worldsheet field, $\widehat{\Phi}_{a a}$ is an antichiral worldsheet field, and $\widehat{\Phi}_{a c}$ is a twisted-antichiral worldsheet field. So the Fradkin-Tseytlin term of (3.5) is worldsheet supersymmetric as desired. Furthermore, one can check that it contains the usual term $\int d z d \bar{z} \varphi r$ where $\varphi$ is the spacetime dilaton and $r$ is the worldsheet curvature.

\subsection{Worldsheet $N=(2,2)$ superconformal invariance}

Although the action of (3.1) is classically worldsheet conformally invariant, $\alpha^{\prime}$ quantum corrections from sigma model loops will spoil this symmetry. After adding contributions from the Fradkin-Tseytlin term of (3.5) and putting the background on-shell, quantum conformal invariance is expected to be restored. However, unlike the bosonic string sigma model, vanishing of the $\beta$-functions is not strong enough to imply the complete set of low-energy equations of motion for the background. This can be seen from a linearized expansion of the sigma model action into the free-field action in a flat background plus the massless closed superstring vertex operator. One can easily see that conformal invariance only implies that the vertex operator is annihilated by $T_{0}$ and $\bar{T}_{0}$, but does not imply the primary field conditions associated with $G^{ \pm}$and $\bar{G}^{ \pm}$.

One therefore expects that quantum worldsheet $N=(2,2)$ superconformal invariance of the sigma model must be imposed in order to obtain the complete set of torsion constraints and low-energy equations of motion for the background superfields. Since worldsheet supersymmetry is not manifest in the hybrid formalism, the simplest way to verify worldsheet $N=(2,2)$ superconformal invariance is to check that the worldsheet $N=(2,2)$ superconformal generators in a curved background satisfy the correct OPE's.

For the hybrid formalism in a four-dimensional background, these OPE's were explicitly computed at tree-level (for heterotic and Type II compactifications) and one-loop (for heterotic compactifications), and shown to imply the correct torsion constraints and low-energy equations of motion for the background superfields. Since the sigma model action in a four-dimensional background closely resembles the action of (3.1) and (3.5), one expects this will also be true in a two-dimensional background.

In a curved background described by (3.1) and (3.5), the worldsheet $N=(2,2)$ superconformal generators are defined as

$$
T=\Pi_{\ddagger} \Pi_{=}-d_{+} \Pi^{+}-d_{\dot{+}} \Pi^{\dot{+}}+\left(\partial \omega-A_{M}^{L} \partial Z^{M}\right)^{2}-\left(\partial \rho-A_{M}^{1} \partial Z^{M}\right)^{2}
$$




$$
\begin{aligned}
& -\partial\left(\partial \omega-A_{M}^{L} \partial Z^{M}\right)+T_{G S}+\alpha^{\prime} \partial^{2}\left(\widehat{\Phi}_{c c}+\widehat{\Phi}_{a a}+\widehat{\Phi}_{c a}+\widehat{\Phi}_{a c}\right) \\
& G^{-}=d_{+} e^{w-\rho}+G_{G S}^{-}+G_{-\frac{1}{2}}^{-} \partial\left(\widehat{\Phi}_{c c}+\widehat{\Phi}_{c a}\right), \\
& G^{+}=e^{\oint \Pi=e^{-2 \omega}}\left(d_{\dot{+}} e^{w+\rho}\right) e^{-\oint \Pi=e^{-2 \omega}}+G_{G S}^{+}+G_{-\frac{1}{2}}^{+} \partial\left(\widehat{\Phi}_{a a}+\widehat{\Phi}_{a c}\right), \\
& J=-2 \partial \rho+J_{G S}+\partial\left(\widehat{\Phi}_{c c}-\widehat{\Phi}_{a a}+\widehat{\Phi}_{c a}-\widehat{\Phi}_{a c}\right) \\
& \bar{T}=\bar{\Pi}_{\ddagger} \bar{\Pi}_{=}-d_{-} \bar{\Pi}^{-}-d_{-} \bar{\Pi}^{\dot{-}}+\left(\bar{\partial} \bar{\omega}+A_{M}^{L} \bar{\partial} Z^{M}\right)^{2}-\left(\bar{\partial} \rho-A_{M}^{2} \partial Z^{M}\right)^{2} \\
& -\bar{\partial}\left(\bar{\partial} \omega+A_{M}^{L} \bar{\partial} Z^{M}\right)+\bar{T}_{G S}+\alpha^{\prime} \bar{\partial}^{2}\left(\widehat{\Phi}_{c c}+\widehat{\Phi}_{a a}+\widehat{\Phi}_{c a}+\widehat{\Phi}_{a c}\right) \\
& \bar{G}^{-}=d_{-} e^{\bar{w}-\bar{\rho}}+\bar{G}_{G S}^{-}+\bar{G}_{-\frac{1}{2}}^{-} \bar{\partial}\left(\widehat{\Phi}_{c c}+\widehat{\Phi}_{a c}\right),
\end{aligned}
$$

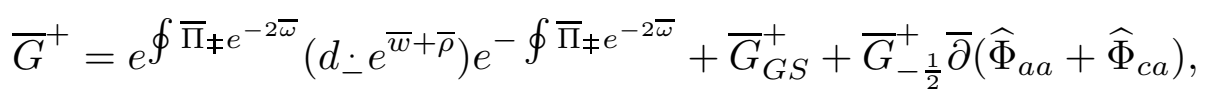

$$
\begin{aligned}
& \bar{J}=-2 \bar{\partial} \bar{\rho}+\bar{J}_{G S}+\bar{\partial}\left(\widehat{\Phi}_{c c}-\widehat{\Phi}_{a a}-\widehat{\Phi}_{c a}+\widehat{\Phi}_{a c}\right) .
\end{aligned}
$$

Note that the Fradkin-Tseytlin contribution to the constraints of (3.8) are analogous to those discussed in [6] and [30] for the four-dimensional background.

\subsection{Torsion constraints and conformal compensators}

Although no explicit calculations will be done here, it is easy to generalize the results of [6] to determine the superspace torsion constraints which come from imposing tree-level worldsheet superconformal invariance of the sigma model. Using the results of [6] and reducing the target-space indices from four-dimensional superspace to two-dimensional superspace, one obtains the superspace torsion constraints

$$
\begin{gathered}
Y_{c c}=T_{\dot{+}=}^{-}=T_{\dot{-} \neq}^{+}, \quad Y_{a a}=T_{+=}^{\dot{\dot{ }}}=T_{-\ddagger}^{\dot{+}}, \\
Y_{c a}=T_{\dot{+}=}^{\dot{\dot{ }}}=T_{-\ddagger}^{+}, \quad Y_{a c}=T_{+=}^{-}=T_{\dot{-\ddagger}}^{\dot{\dot{q}}}, \\
T_{+\dot{+}}^{\ddagger}=T_{-\dot{-}}^{=}=H_{\neq-\dot{-}}=H_{=+\dot{+}}=1,
\end{gathered}
$$

where $T_{A B}^{C}$ is the torsion defined using $\left[\nabla_{A}, \nabla_{B}\right]=T_{A B}^{C} \nabla_{C}+\ldots$ and $H_{A B C}=\nabla_{[A} B_{B C]}+$ $T_{[A B}^{D} B_{C] D}$ is the tensor field strength.

The first two lines of (3.10) imply that the R-R field strengths are contained in the supergravity multiplet and the third line of (3.10) implies that spacetime conformal invariance of the background superfields has been broken by fixing $H_{\neq--}$to be a constant. 
Note that Bianchi identities imply that all components of $H_{A B C}$ are determined by a scalar superfield $G$ satisfying $H_{\ddagger-\dot{-}}=H_{=+\dot{+}}=G$. However, if the target-space theory is made conformally invariant by including conformal compensator superfields, one can fix conformal invariance by gauging $G=1$.

As in the four and six-dimensional hybrid formalisms, exponentials of the chiral and twisted-chiral spacetime superfields appearing in the Fradkin-Tseytlin term of (3.5) will play the role of the $U(1) \times U(1)$ conformal compensator superfields. These compensator superfields are convenient for constructing superspace actions for supergravity [31. Under conformal and $U(1) \times U(1) R$ transformations parameterized by the superfields $L, R^{1}$ and $R^{2}$, the logarithm of the compensator superfields will be defined to transform as

$$
\begin{gathered}
\delta \Phi_{c c}=i\left(R_{1}+R_{2}\right)+L, \quad \delta \Phi_{c a}=i\left(R_{1}-R_{2}\right)+L, \\
\delta \Phi_{a c}=i\left(-R_{1}+R_{2}\right)+L, \quad \delta \Phi_{a a}=i\left(-R_{1}-R_{2}\right)+L .
\end{gathered}
$$

Since the torsion constraints of (3.10) break conformal invariance by fixing $H_{\neq--}$to a constant, the real part of $\Phi_{c c}+\Phi_{c a}$ is an independent degree of freedom whose lowest component is the spacetime dilaton. But since spacetime $U(1) \times U(1) R$ symmetry is unbroken by the torsion constraints, the imaginary part of $\Phi_{c c}+\Phi_{c a}$ and $\Phi_{c c}+\Phi_{a c}$ are gauge degrees of freedom which can be fixed to zero.

\subsection{Superspace effective action}

As in the four-dimensional hybrid formalism, the low-energy superspace effective action can be constructed in a spacetime conformally invariant manner from the superfields $E_{A}^{M}, G$ and $e^{\Phi}$ which were discussed in the previous subsection. In Einstein gauge, one breaks conformal invariance by gauge-fixing $e^{\Phi}=1$ which produces a component action of the form $S=\int d^{D} x[\sqrt{g} R+\ldots]$. But in string gauge, one breaks conformal invariance by gauge-fixing $G=1$ which produces a component action of the form $S=\int d^{D} x e^{-2 \varphi}[\sqrt{g} R+\ldots]$. The low-energy effective action coming from the sigma model is in string gauge since the dilaton coupling in the Fradkin-Tseytlin term implies that all NS-NS terms appear with a factor of $e^{-2 \varphi}$.

As in the four-dimensional and ten-dimensional Type II effective actions, the kinetic term for the R-R gauge fields, $\left(d A_{R-R}\right)^{2}$, appears with no $e^{-2 \varphi}$ factor. The explanation 
of this fact [6] is that the R-R field strength $d A_{R-R}$ appears in the sigma model through the $(\theta)^{2}$ component of the compensator superfields $e^{-\Phi}$, i.e.

$$
\begin{gathered}
e^{-\Phi_{c c}}=e^{-\phi_{c c}}+\theta^{+} \theta^{-} F_{+-}+\ldots, \quad e^{-\Phi_{a a}}=e^{-\phi_{a a}}+\theta^{\dot{\dot{+}}} \theta^{\dot{-}} F_{\dot{+}-}+\ldots, \\
e^{-\Phi_{c a}}=e^{-\phi_{c a}}+\theta^{+} \theta^{\dot{-}} F_{+\dot{-}}+\ldots, \quad e^{-\Phi_{a c}}=e^{-\phi_{a c}}+\theta^{\dot{+}} \theta^{-} F_{\dot{+}-}+\ldots,
\end{gathered}
$$

where $F_{\alpha \beta}$ is defined in (2.20). Of the four scalars appearing in (3.12), the dilaton $\varphi$ is identified with $\frac{1}{2} \operatorname{Re}\left(\phi_{c c}+\phi_{c a}\right) \cdot \operatorname{Im}\left(\phi_{c c}\right)$ and $\operatorname{Im}\left(\phi_{c a}\right)$ can be gauged away using the $U(1) \times U(1)$ gauge invariance, and the remaining scalar, $\operatorname{Re}\left(\phi_{c c}-\phi_{c a}\right)$, will vanish onshell. Note that in four dimensions, (3.12) implies that $e^{-\Phi}$ must satisfy certain Bianchi identities, but these identities are absent in two dimensions since the field strengths are scalars. In two dimensions, the field strength superfield for a vector multiplet is obtained by simply dualizing the auxiliary scalar(s) in a chiral or twisted-chiral superfield [32].

Ignoring the CY moduli, the low-energy superspace action is expected to be

$$
S=\int d^{2} x \int d \theta^{+} d \theta^{-} d \theta^{\dot{+}} d \theta^{\dot{-}}(\operatorname{sdet} E)\left(e^{-\Phi_{c c}-\Phi_{a a}}-e^{-\Phi_{c a}-\Phi_{a c}}\right) .
$$

As in four dimensions, $\int d^{4} \theta e^{-2 \Phi}$ produces $F^{2}$ terms with no $e^{-2 \varphi}$ factor as can be seen from the component expansion of (3.12). And the R-R component fields which appear in the lowest component of $\left[Y_{c c}, Y_{c a}, Y_{a c}, Y_{a a}\right]$ of (3.10) are actually auxiliary fields $y_{\alpha \beta}$ which are related to the R-R field strengths $F_{\alpha \beta}$ by the equations of motion $y_{\alpha \beta}=e^{\varphi} F_{\alpha \beta}$. Up to proportionality constants, the compactification-independent bosonic contribution to the low-energy effective action is

$$
\begin{aligned}
S= & \int d^{2} x \sqrt{-g}\left[e^{-2 \varphi}\left(R+4 \nabla_{\ddagger} \varphi \nabla_{=} \varphi+y_{+-} y_{\dot{+} \dot{-}}+y_{\dot{+}-} y_{+\dot{-}}\right)\right. \\
& -e^{-\varphi}\left(y_{+-} F_{\dot{+}-\dot{ }}+y_{\dot{+}-} F_{+-}+y_{+\dot{-}} F_{\dot{+}-}+y_{\dot{+}-} F_{+\dot{+}}\right) \\
& \left.-F_{+-} F_{\dot{+}-}-F_{+\dot{-}} F_{\dot{+}-}+d\left(e^{-\phi_{c c}-\phi_{a a}}-e^{-\phi_{c a}-\phi_{a c}}\right)\right],
\end{aligned}
$$

where $\varphi=\frac{1}{2} \operatorname{Re}\left(\phi_{c c}+\phi_{c a}\right)$ and $d(x)$ is an auxiliary field contained in the $\theta^{4}$ component of sdetE. After integrating out $y_{\alpha \beta}$ and $d$,

$$
S=\int d^{2} x \sqrt{-g}\left[e^{-2 \varphi}\left(R+4 \nabla_{\ddagger} \varphi \nabla_{=} \varphi\right)-2 F_{+-} F_{\dot{+}-}-2 F_{+\dot{-}} F_{\dot{+}-}\right] .
$$

In the next section, we shall consider adding to the action a cosmological term proportional to $e^{-2 \varphi}$, i.e. $c \int d^{2} x \sqrt{-g} e^{-2 \varphi}$, which arises when the total matter and ghost central 
charges do not cancel. Such a term can be supersymmetrized by adding the chiral and twisted-chiral F-terms

$$
\begin{aligned}
S^{\prime}= & \int d^{2} x\left[c_{\dot{+} \dot{-}} \int d \theta^{+} d \theta^{-} e^{-2 \Phi_{c c}}+c_{+-} \int d \theta^{\dot{+}} d \theta^{\dot{-}} e^{-2 \Phi_{a a}}\right. \\
& \left.+c_{\dot{+}-} \int d \theta^{+} d \theta^{\dot{-}} e^{-2 \Phi_{c a}}+c_{+\dot{-}} \int d \theta^{\dot{+}} d \theta^{-} e^{-2 \Phi_{a c}}\right]
\end{aligned}
$$

to the action of (3.13). Using the component analysis of (3.12), the bosonic contribution to $S^{\prime}$ is

$$
S^{\prime}=2 \int d^{2} x \sqrt{g} e^{-\varphi}\left(c_{\dot{+} \dot{-}} F_{+-}+c_{+-} F_{\dot{+} \dot{-}}+c_{\dot{+}-} F_{+\dot{-}}+c_{+\dot{-}} F_{\dot{+}-}\right) .
$$

Note that in the presence of $S^{\prime}$, the equations of motion for the R-R field strengths are $\partial_{m}\left(F_{\alpha \beta}-c_{\alpha \beta} e^{-\varphi}\right)=0$. Plugging these auxiliary equations of motion into the action $S+S^{\prime}$, one obtains

$$
S_{e f f}=S+S^{\prime}=\int d^{2} x \sqrt{-g}\left[e^{-2 \varphi}\left(R+4(\nabla \varphi)^{2}+c\right)+\Lambda\right]
$$

where $\Lambda=-2\left(|W|^{2}+|\widetilde{W}|^{2}\right)$ and $c=2\left(\left|c_{+-}\right|^{2}+\left|c_{+-}\right|^{2}\right)$ are constants, and

$W=F_{+-}-c_{+-} e^{-\varphi}, \quad \bar{W}=F_{\dot{+}-}-c_{\dot{+}-} e^{-\varphi}, \quad \widetilde{W}=F_{+\dot{-}}-c_{+\dot{+}} e^{-\varphi}, \quad \widetilde{W}=F_{\dot{+}-}-c_{\dot{+}-} e^{-\varphi}$.

Note that spacetime supersymmetry implies that $c \geq 0$ and $\Lambda \leq 0$.

\section{New Two-Dimensional Black Holes}

In this section we study the effective action of superstrings in two-dimensional backgrounds with Ramond-Ramond flux and "excess" central charge (3.18):

$$
S_{2 d}=\int d^{2} x \sqrt{-g}\left[e^{-2 \varphi}\left(R+4(\nabla \varphi)^{2}+c\right)+\Lambda\right]
$$

Here both the "excess central charge" $c$ and the "cosmological constant" $\Lambda$ have dimension mass squared since we absorbed a factor of $\alpha^{\prime}$ in the definition of $c$.

As we explained above, the generalized dilaton gravity action (4.1) is very natural from the string theory point of view, since it includes both the central charge term $c$ and the cosmological constant term $\Lambda$ corresponding to background Ramond-Ramond fields. In the special limiting cases, corresponding to $\Lambda=0$ and to $c=0$, our action (4.1) reduces, correspondingly, to the CGHS model [11] also inspired by string theory, and to the dilaton 
gravity model studied by Mignemi [33]. General dilaton gravity Lagrangians of the form (4.1) have been considered in the literature, see e.g. [13,34,35, 36, 37, 38. However, explicit solutions and their properties have not been analysed in detail for the specific model (4.1) with two arbitrary parameters $c$ and $\Lambda$. This will be our main goal in this section. Namely, we construct a large family of new static black hole solutions in this model, including extremal solutions for $\Lambda<0$ and Schwarzschild-like black holes for $\Lambda>0$, and discuss their dynamical aspects, such as formation and evaporation.

\subsection{Extremal Black Hole Solution}

Variation of the action (4.1) with respect to the metric and the dilaton lead to the equations of motion:

$$
\nabla_{\mu} \nabla_{\nu} \varphi+g_{\mu \nu}\left((\nabla \varphi)^{2}-\nabla^{2} \varphi-\frac{c}{4}-\frac{\Lambda}{4} e^{2 \varphi}\right)=0
$$

and:

$$
R+c+4 \nabla^{2} \varphi-4(\nabla \varphi)^{2}=0
$$

It is convenient to rewrite these equations of motion in the light-cone coordinates $x^{\ddagger}=$ $x^{0}+x^{1}, x^{=}=x^{0}-x^{1}$, and use the conformal gauge:

$$
g_{\text {キ= }}=-\frac{1}{2} e^{2 \rho}, \quad g_{\text {fキ }}=0, \quad g_{==}=0
$$

In a compact form, this anzatse for the metric can be written as $g_{\mu \nu}=e^{2 \rho} \eta_{\mu \nu}$, where $\eta_{\mu \nu}$ has signature $(-,+)$. In this gauge, the scalar curvature is

$$
R=8 e^{-2 \rho} \partial_{\ddagger} \partial=\rho,
$$

the equation of motion for the metric (4.2) becomes, $c f$. [11,9]:

$$
2 \partial_{\ddagger} \partial=\varphi-4 \partial_{\ddagger} \varphi \partial=\varphi-\frac{c}{4} e^{2 \rho}-\frac{\Lambda}{4} e^{2(\varphi+\rho)}=0,
$$

and the dilaton equation of motion (4.3) reads:

$$
-2 \partial_{\ddagger} \partial=\varphi+2 \partial_{\ddagger} \varphi \partial_{=} \varphi+\partial_{\ddagger} \partial=\rho+\frac{c}{8} e^{2 \rho}=0 .
$$

Since we have gauge fixed $g_{\neq \neq}$and $g_{==}$to zero we must also impose their equations of motion as constraints:

$$
2 \partial_{\ddagger} \rho \partial_{\ddagger} \varphi-\partial_{\ddagger}^{2} \varphi=0
$$


and

$$
2 \partial=\rho \partial=\varphi-\partial_{=}^{2} \varphi=0
$$

We are interested in static solutions. Therefore, we assume that $\rho(x)$ and $\varphi(x)$ depend only on the spatial coordinate $x \equiv x^{1}=\left(x_{\ddagger}-x_{=}\right) / 2$. This gives:

$$
\begin{gathered}
\varphi^{\prime \prime}-2 \varphi^{\prime 2}+\frac{c}{2} e^{2 \rho}+\frac{\Lambda}{2} e^{2(\varphi+\rho)}=0 \\
-2 \varphi^{\prime \prime}+2 \varphi^{\prime 2}+\rho^{\prime \prime}-\frac{c}{2} e^{2 \rho}=0 \\
\varphi^{\prime \prime}-2 \varphi^{\prime} \rho^{\prime}=0
\end{gathered}
$$

for the equations of motion (4.5), (4.6), and for the constraints (4.7) - (4.8), respectively.

The last equation can be easily solved to give a relation between the conformal factor and the dilaton:

$$
e^{2 \rho}=A \varphi^{\prime}
$$

for some non-zero constant $A$. By a suitable choice of the coordinate $x$ we can always set $A=1$. Notice that the sign of $A$ is related to the orientation of our solution in one-dimensional space.

Substituting into (4.9) we find:

$$
\varphi^{\prime \prime}-2 \varphi^{\prime 2}=-\frac{c}{2} \varphi^{\prime}-\frac{\Lambda}{2} e^{2 \varphi} \varphi^{\prime}
$$

whose first integral is:

$$
\varphi^{\prime} e^{-2 \varphi}-\frac{c}{4} e^{-2 \varphi}+\frac{\Lambda}{2} \varphi+m=0
$$

As we explain in the following, this solution describes a two-dimensional black hole, and the integration constant $m$ is proportional to the mass of the black hole. Using the relation (4.12) we can write down the solution for conformal factor of the metric as a function of the dilaton:

$$
e^{2 \rho}=\varphi^{\prime}=\frac{c}{4}-e^{2 \varphi}\left(\frac{\Lambda}{2} \varphi+m\right)
$$

It can be integrated further to give an expression for the dilaton as a function of the coordinate $x$ (which is defined up to a shift):

$$
x=-\frac{1}{2} \int_{-\infty}^{-2 \varphi} \frac{e^{z} d z}{\left(\frac{c}{4} e^{z}+\frac{\Lambda}{4} z-m\right)}
$$


At the infinity, $x \rightarrow-\infty$, the dilaton field, given by the inverse of this function, grows linear with $x$ :

$$
\varphi(x) \approx \frac{c}{4} x-\frac{2}{c} e^{\frac{c}{2} x}\left(\frac{c \Lambda}{8} x+m-\frac{\Lambda}{4}\right)+\ldots
$$

while the metric $g_{\mu \nu} \approx \frac{c}{4} \eta_{\mu \nu}$ is asymptotically flat. Therefore, at one spatial infinity our solution looks like a linear dilaton background, $c f$. [11]. It turns out that the dilaton is exactly linear in a Schwarzschild-like gauge, where the solution (4.15) looks like:

$$
d s^{2}=-l(\varphi) d t^{2}+\frac{1}{l(\varphi)} d \varphi^{2}
$$

and the dilaton $\varphi$, which plays a role of a spatial coordinate here, is related to the tortoise coordinate $x$ as $d \varphi=l(\varphi) d x$. If we compare this relation with (4.12), we find that $l(\varphi)$ is nothing but the conformal factor expressed in terms of the dilaton (4.15):

$$
l(\varphi)=\frac{c}{4}-e^{2 \varphi}\left(\frac{\Lambda}{2} \varphi+m\right)
$$

The Schwarzschild gauge is very convenient, so for the rest of this section we shall work in this gauge with the solution (4.17) - 4.18).

Now we discuss the physical properties of this solution. First, we compute the scalar curvature (4.4):

$$
R=-2 e^{-2 \rho} \rho^{\prime \prime}
$$

which in the Schwarzschild gauge has a very simple form:

$$
R=-\frac{d^{2} l(\varphi)}{d \varphi^{2}}=-2 e^{2 \varphi}(\Lambda \varphi+2 m+\Lambda)
$$

We find that $R$ goes to zero at minus infinity, $\varphi \rightarrow-\infty$, in agreement with our earlier observation, and diverges at the plus infinity, $\varphi \rightarrow+\infty$, where string perturbation theory breaks down.

The points in space where $l(\varphi)=0$ describe the horizon manifold:

$$
\frac{c}{4}-e^{2 \varphi}\left(\frac{\Lambda}{2} \varphi+m\right)=0
$$

The position and the number of such points depends on the values of the central charge $c$ and the cosmological constant $\Lambda$. For our solution to make sense at $\varphi \rightarrow-\infty$, where string perturbation theory is valid, we have to assume that $c$ is positive, $c f$. (4.15). Then, there are two possibilities corresponding to $\Lambda>0$ and $\Lambda<0$, shown on Fig.1. 


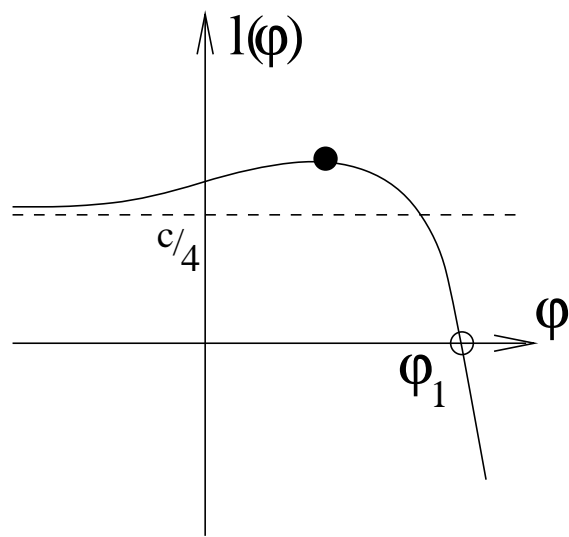

a)

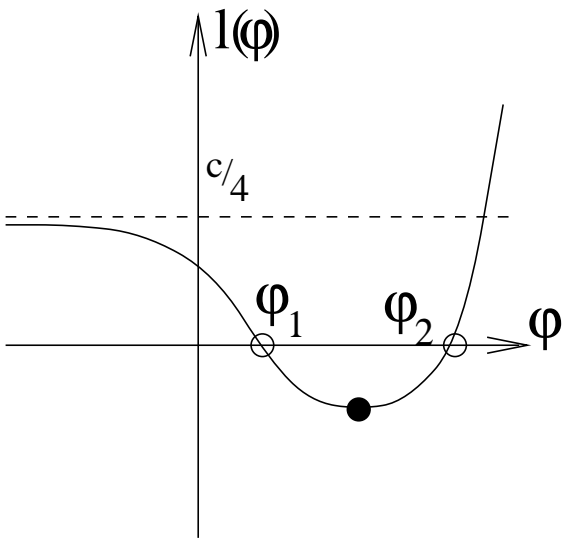

b)

Fig. 1: A plot of the conformal factor for black hole solutions $a$ ) with $\Lambda>0$, and b) with $\Lambda<0$.

In the first case $(\Lambda>0)$ there is always one horizon, and the solution (1.18) looks like a Schwarzschild black hole, see the corresponding Penrose diagram on Fig.2a. On the other hand, a general non-singular solution with $\Lambda<0$ has two horizons, at $\varphi_{1}$ and $\varphi_{2}$, just like the usual Reissner-Nordstrom black hole in four dimensions. Similar to the ReissnerNordstrom solution, we have two conformal blocks for each of the intervals $\left(-\infty, \varphi_{1}\right)$, $\left(\varphi_{1}, \varphi_{2}\right)$, and $\left(\varphi_{2},+\infty\right)$, which altogether represent a fundamental region for the twodimensional analog of the Reissner-Nordstrom black hole, see the corresponding Penrose diagram on Fig.2b.

As we will see in a moment, extremal solutions can appear only if $\Lambda<0$ in agreement with our expectations from string theory, which also suggests the existence of extremal solutions only for negative values of the cosmological constant. Before we demonstrate this, let us briefly comment on the geodesic completeness across a horizon, $c f$. [39,40. For a static solution the completeness of space-like geodesics follows from the equation:

$$
\lim _{\varphi \rightarrow \varphi_{h}} t \rightarrow \int^{\varphi_{h}} \frac{d \varphi}{l(\varphi)}
$$

For our solutions (4.18), this integral always diverges at a horizon corresponding to finite value of $\varphi$, where $l(\varphi)$ has a simple zero (or zero of order two in the extremal case).

An extremal black hole appears when two horizons coincide, i.e. when the function $l(\varphi)$ has a double zero at some point $\varphi=\varphi_{0}$. As we mentioned above, this may occur only for negative cosmological constant, $\Lambda<0$. In fact, to determine the value of $\varphi_{0}$, we 


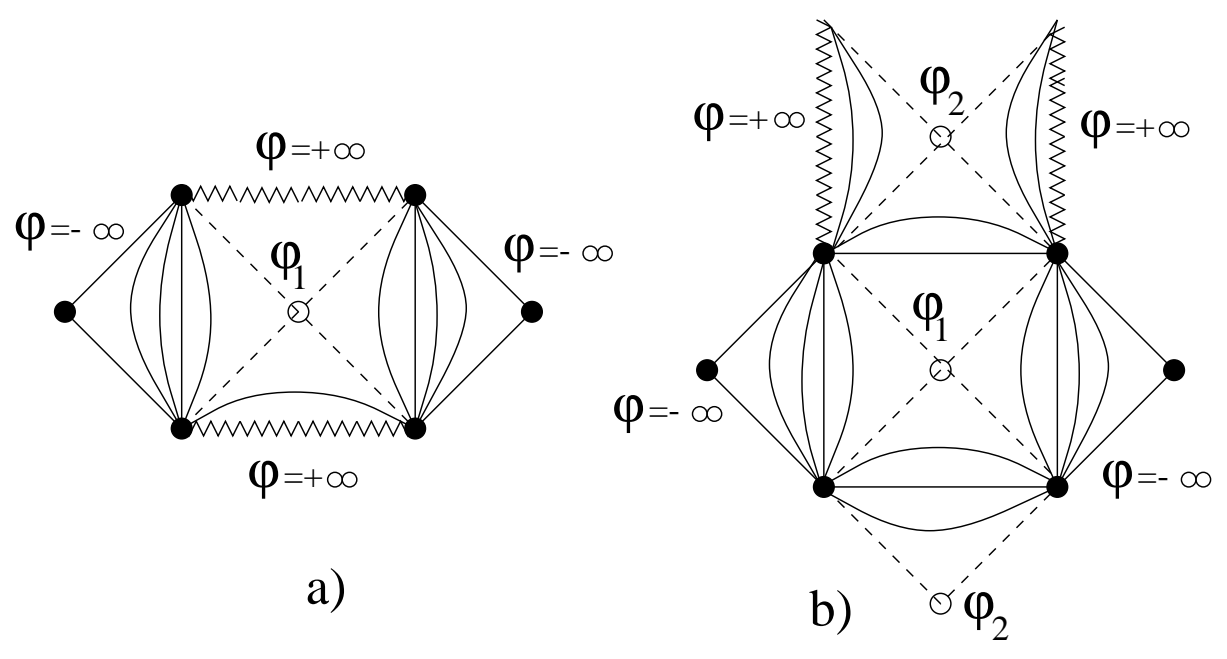

Fig. 2: Penrose diagram a) for a Schwarzschild-like two-dimensional black hole $(\Lambda>0)$, and $b)$ for a two-dimensional analog of the Reissner-Nordstrom black hole $(\Lambda<0)$. Values of the dilaton are indicated at the horizons and at the asymptotic regions.

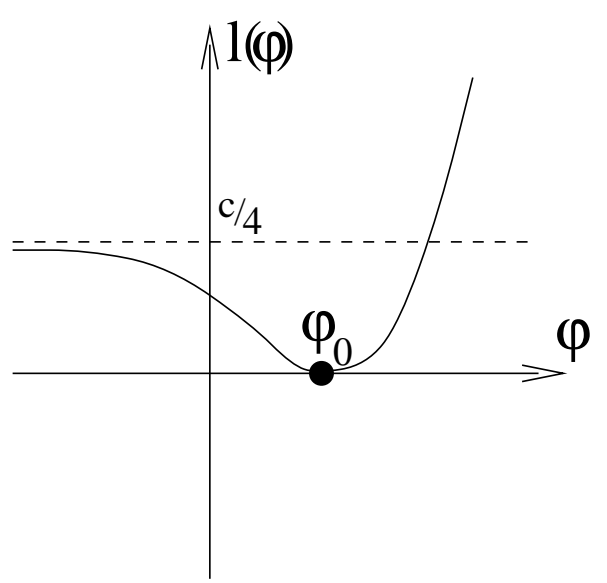

a)

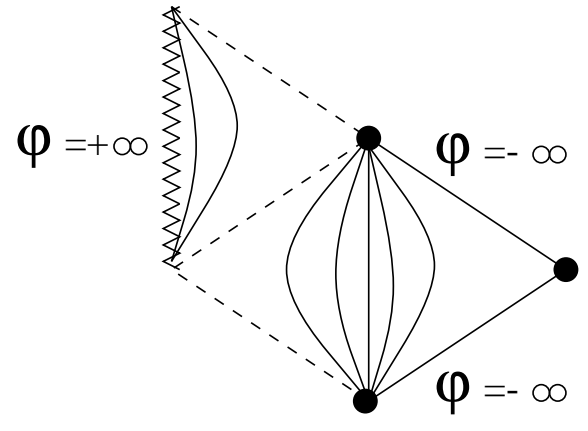

b)

Fig. 3: Conformal factor $(a)$ and the Penrose diagram $(b)$ for the extremal black hole solution. Only one fundamental region is shown on the Penrose diagram.

have to solve equations: $l(\varphi)=0$ and $l^{\prime}(\varphi)=0$. Therefore, along with (4.19) we have an equation:

$$
e^{2 \varphi}\left(\Lambda \varphi+2 m+\frac{\Lambda}{2}\right)=0
$$

which has the solution:

$$
\varphi_{0}=-\frac{2 m}{\Lambda}-\frac{1}{2}
$$

Substituting this into (4.19), we get the extremality condition (BPS condition) for our 
black hole solution:

$$
m_{0}=-\frac{\Lambda}{4}-\frac{\Lambda}{4} \ln \left(-\frac{c}{\Lambda}\right) .
$$

Now it is clear that for positive $c$ this expression makes sense only if the cosmological constant $\Lambda$ is negative. The conformal factor and Penrose diagram (that now consists of two symmetric fundamental regions) for the extremal black hole are shown in Fig.3. The solution (4.18) has a naked singularity unless the mass parameter obeys the BPS inequality $m \geq m_{0}$.

We can calculate the geometric Hawking temperature as the normal derivative of the norm of the Killing vector $\partial / \partial t$ at the horizon. In the Schwarzschild gauge it has a very simple form:

$$
T_{H}=\frac{1}{2}\left|\frac{d l(\varphi)}{d \varphi}\right|_{h}
$$

From the explicit expression (4.18) for the function $l(\varphi)$ it is easy to see that $T_{H}$ is equal to zero precisely when black becomes extremal, i.e. when both eqns. (4.19) and (4.20) hold. Therefore, in quantum theory coupled to matter, non-extremal black holes (4.17) - (4.18) are expected to emit Hawking radiation until they reach the endpoint of evaporation - the extremal black hole with $m=m_{0}$, see [34] and below. Furthermore, non-extremal solutions with $m>m_{0}$ are expected to be unstable against instanton-mediated fragmentation into disconnected universes [41].

On the other hand, the extremal solutions are expected to be stable and $1 / 2$ BPS in the appropriate supersymmetric theory. Although all of the black hole solutions (4.17) constructed here represent supersymmetric ground states in $\mathcal{N}=1$ dilaton supergravity [36] with the dilaton superpotential:

$$
\mathcal{W}(\Phi)=e^{-\Phi} \sqrt{c e^{-2 \Phi}-2 \Lambda \Phi-4 m}
$$

only extremal solutions are expected to correspond to BPS saturated states in the extended $\mathcal{N}=2$ supergravity. Note that in the limit $\Lambda \rightarrow 0$, the mass of the extremal solution also goes to zero, according to (4.22), and the solution degenerates into a linear dilaton background.

Note also that in the near horizon limit, the extremal black hole solution (4.17) looks like two-dimensional anti-de Sitter space. For the extremal solution, $l\left(\varphi_{0}\right)=0$ and $l^{\prime}\left(\varphi_{0}\right)=$ 0 , so that to the leading order in $\left(\varphi-\varphi_{0}\right)$ we have:

$$
l(\varphi) \approx \frac{c}{2}\left(\varphi-\varphi_{0}\right)^{2} .
$$


In terms of the new space-like variable $u^{-1}=\sqrt{\frac{c}{2}}\left(\varphi-\varphi_{0}\right)$ the metric takes a more standard form:

$$
d s^{2}=\frac{1}{u^{2}}\left(-d t^{2}+R_{A d S}^{2} d u^{2}\right)
$$

where $R_{A d S}=\sqrt{\frac{2}{c}}$ is the characteristic scale parameter.

\subsection{Mass of the Black Hole}

Note that $m$ has dimension of mass squared, so $m / \sqrt{c}$ is expected to be related to the physical mass parameter up to some numerical factor. The appropriate formula for the definition of the ADM mass in generalized two-dimensional dilaton gravity was proposed by Mann [42], and also in a recent work [43]. Below we show that both methods lead to the same answer for the ADM mass:

$$
M_{A D M}=\frac{2}{\sqrt{c}} m
$$

According to [43], the ADM mass of a solution like (4.17) in a generalized dilaton gravity theory can be obtained evaluating the following expression at the spatial infinity 6 :

$$
M_{A D M}-M_{0}=\frac{4}{\sqrt{c}} e^{-2 \varphi}\left[\sqrt{l(\varphi) l_{0}(\varphi)}-l(\varphi)\right]_{\varphi \rightarrow-\infty}
$$

where the index 0 refers to the reference solution, that we choose to be the extremal solution with $m=m_{0}, c f$. (4.22). Substituting (4.18) into (4.26), we find:

$$
\begin{aligned}
& M_{A D M}-M_{0}=\frac{e^{-2 \varphi}}{\sqrt{c}} {\left[\sqrt{\left(c-2 e^{2 \varphi}\left(\Lambda \varphi+2 m_{0}\right)\right)\left(c-2 e^{2 \varphi}(\Lambda \varphi+2 m)\right)}\right.} \\
&\left.-\left(c-2 e^{2 \varphi}(\Lambda \varphi+2 m)\right)\right]_{\varphi \rightarrow-\infty}
\end{aligned}
$$

Evaluating this expression in the limit $\varphi \rightarrow-\infty$, we get (4.25).

To provide further evidence that, up to a universal numerical factor, the mass of the black hole is given by $m$, we use the general formulae proposed by Mann [42], that in our case reads, $c f$. [13]:

$$
\begin{aligned}
E_{A D M} & =\frac{1}{2 \sqrt{c}}\left[e^{-2 \varphi}\left(-4(\nabla \varphi)^{2}+c\right)+\Lambda\right]_{\varphi \rightarrow \infty} \\
& =\frac{1}{2 \sqrt{c}}\left[e^{-2 \varphi}(-4 l(\varphi)+c)+\Lambda\right]_{\varphi \rightarrow \infty} \\
& =\frac{1}{2 \sqrt{c}}[2 \Lambda \varphi+4 m+\Lambda]_{\varphi \rightarrow \infty}
\end{aligned}
$$

8 The sign and normalization in this formulae are chosen in such a way to agree with our earlier conventions, $c f$. (4.12). 
Once appropriately regularized, this expression clearly leads to (4.25):

$$
E_{A D M}(m)-E_{A D M}\left(m=m_{0}\right)=\frac{2}{\sqrt{c}}\left(m-m_{0}\right)
$$

Similar to the Reissner-Nordstrom solution, our solution (4.18) develops a naked singularity for $m<m_{0}$. Therefore, a condition that a given black hole does not have such singularities can be expressed in a form of the BPS inequality:

$$
M \geq M_{0}
$$

where $M_{0}$ is given by $(4.22)$ :

$$
M_{0}=-\frac{\Lambda}{2 \sqrt{c}}\left(1+\ln \left(-\frac{c}{\Lambda}\right)\right)
$$

Note, that for $\Lambda<0$ this expression is always positive.

\subsection{The Limit $\Lambda \rightarrow 0$}

In the limit $\Lambda \rightarrow 0$, the action (4.1) looks like the usual string-inspired dilaton gravity action:

$$
L=\int d^{2} x \sqrt{-g} e^{-2 \varphi}\left(R+4(\nabla \varphi)^{2}+c\right)
$$

which, in the Euclidean signature, has a semi-infinite cigar solution 9 [8,9,10]:

$$
\begin{aligned}
d s^{2} & =\frac{k}{2}\left(d r^{2}+\tanh ^{2}(r) d \psi^{2}\right) \\
\varphi & =-\log \cosh (r)+\varphi_{0}
\end{aligned}
$$

where $k$ is related to the central charge $c$ as follows:

$$
c=\frac{8}{k-2}=\frac{8}{k^{\prime}}
$$

Hence, it is natural to expect that in this limit our solution (4.17) looks like a cigar solution, more precisely, like its analog in the Minkowski signature obtained by a Wick rotation $\psi \rightarrow i t$ [10]:

$$
d s^{2}=\frac{k}{2}\left(d r^{2}-\tanh ^{2}(r) d t^{2}\right)
$$

\footnotetext{
9 Our normalization for the dilaton field in the action (4.1) is different from the normalization used in $8,9,10]$. The notations differ by a factor of $2 ; \Phi=2 \varphi$.

10 Note, in our notations, $c$ is the 'excess central charge,' rather than the central charge of the $S L(2, R) / U(1)$ gauged WZW model, denoted in [10] by the same letter.
} 
Note, that the $U(1)$ isometry of the cigar solution, generated by shifts of the angle variable $\psi$ becomes the invariance with respect to time translations in the Minkowski signature.

Expressed in terms of the new space-like coordinate $\rho=\log \cosh (r)$ and in terms of the rescaled time-like variable $t \rightarrow(2 / k) t$, the metric (4.35) indeed looks like the non-extremal solution (4.17) with zero cosmological constant, $\Lambda=0$ :

$$
d s^{2}=-l(\rho) d t^{2}+\frac{1}{l(\rho)} d \rho^{2}
$$

where:

$$
l(\rho)=\frac{2}{k}\left(1-e^{-2 \rho}\right)
$$

From (4.33), we also find the dilaton $\varphi=-\rho+\varphi_{0}$, which, in agreement with our solution (4.17), turns out to be linear. In terms of $\varphi$, the metric (4.36) is identical to our metric (4.17) with $\Lambda=0$ and $l(\varphi)$ given by:

$$
l(\varphi)=\frac{2}{k}-\frac{2 e^{-2 \varphi_{0}}}{k} e^{2 \varphi}
$$

If we compare this expression with (4.18), we can identify the parameters as follows:

$$
\frac{c}{4}=\frac{2}{k}
$$

and:

$$
m=\frac{2}{k} e^{-2 \varphi_{0}}
$$

The relation (4.38) between the central charge $c$ and the level $k$, in the semiclassical approximation, is clearly compatible with the exact formula (4.34). Furthermore, if we start with zero cosmological constant, $\Lambda=0$, the string one-loop amplitude (i.e. the torus partition function) is expected to generate a non-zero $\Lambda$-term 1 . A back reaction of this perturbation to gravity should lead to modification of the cigar geometry [44] and, in particular, to bifurcation of the horizon, as discussed in this section.

The second relation (4.39) that we found comparing the two solutions (4.18) and (4.37) explains the interpretation of the parameter $m$ as the mass of the black hole. Indeed, according to [10], the mass of the classical cigar solution (4.35) is given by:

$$
M=\sqrt{\frac{2}{k^{\prime}}} e^{-2 \varphi_{0}}=\frac{2}{\sqrt{c}} m,
$$

which is precisely the ADM mass formula (4.25).

11 We would like to acknowledge Steven Shenker for this comment. 


\subsection{Dynamical Formation of the Near-Extremal Black Hole}

By analogy with the CGHS case [11], we expect that any perturbation of the extremal solution with critical mass $M_{0}$ will result in a formation of the near-extremal solution with $M>M_{0}$, where $M_{0}$ is given by (4.22):

$$
M_{0}=-\frac{\Lambda}{2 \sqrt{c}}\left(1+\ln \left(-\frac{c}{\Lambda}\right)\right)
$$

In this subsection we explicitly describe the formation process from interaction of the dilaton gravity with a conformal matter field $f$ :

$$
L=\int d^{2} x \sqrt{-g}\left[e^{-2 \varphi}\left(R+4(\nabla \varphi)^{2}+c\right)+\Lambda-\frac{1}{2}(\nabla f)^{2}\right]
$$

Since the solution (4.18) was constructed in such a way that the weak coupling region is at $x=-\infty$, we want to set up the initial conditions accordingly. A convenient choice of the field $f$ that meets this requirement is where $f\left(x^{=}\right)$propagates from the far past along the $x^{\ddagger}$ light-cone direction. To be specific, we choose $f\left(x^{=}\right)$in the form of the shock wave:

$$
\frac{1}{2}\left(\partial_{=} f\right)^{2}=m_{f} \delta\left(x^{=}-x_{0}^{\bar{z}}\right)
$$

In a similar way, we could consider an arbitrary field $f\left(x^{=}\right)$propagating along $x^{\ddagger}$ and still be able to solve the model. It would be interesting to study integrability of the action (4.42) in more detail, following the ideas of [45,46].

Since the matter field $f\left(x^{=}\right)$is assumed to be independent on $x^{\ddagger}$, the constraint (4.7) has the same form as in the theory with $f=0$. As before, we can easily solve this constraint 12 :

$$
e^{2 \rho}=2 \partial_{\neq} \varphi
$$

Then, the remaining constraint and equations of motion that follow from the modified action (4.42) can be written in the following form, $c f$. [43]:

$$
\begin{gathered}
\frac{1}{2}\left(\partial_{=} \varphi\right) \partial_{=}\left[\ln \left(\partial_{=} \varphi\right)-\ln \left(\partial_{\ddagger} \varphi\right)\right]=\frac{\left(f^{\prime}\right)^{2}}{8} e^{2 \varphi} \\
2 \partial_{\ddagger} \partial_{=} \varphi-4 \partial_{\ddagger} \varphi \partial_{=} \varphi-\frac{c}{2}\left(\partial_{\ddagger} \varphi\right)-\frac{\Lambda}{2}\left(\partial_{\ddagger} \varphi\right) e^{2 \varphi}=0
\end{gathered}
$$

12 The numerical factor 2 is chosen to agree with our earlier conventions. 


$$
\partial_{\ddagger} \partial_{=}\left(\varphi-\frac{1}{2} \ln \left(\partial_{\ddagger} \varphi\right)\right)=-\frac{\Lambda}{4}\left(\partial_{\neq} \varphi\right) e^{2 \varphi}
$$

Since the last equation has the form $\partial_{\ddagger}(\ldots$.$) , it can be easily integrated to give:$

$$
\partial_{=}\left(\varphi-\frac{1}{2} \ln \left(\partial_{\mp} \varphi\right)\right)=-\frac{\Lambda}{8} e^{2 \varphi}+\eta\left(x^{=}\right)
$$

where $\eta\left(x^{=}\right)$is some function of $x^{=}$, which will be determined later. Integrating one more time, we get:

$$
\partial_{=} e^{-2 \varphi}+\frac{\Lambda}{2} \varphi+2 e^{-2 \varphi} \eta\left(x^{=}\right)+\lambda\left(x^{=}\right)=0
$$

We want to compare this result to the equation, which follows from (4.46), $c f .(4.14)$ :

$$
\partial=e^{-2 \varphi}-\frac{c}{4} e^{-2 \varphi}+\frac{\Lambda}{2} \varphi+m\left(x^{=}\right)=0
$$

One can immediately identify the integration functions as follows:

$$
\eta\left(x^{=}\right)=-\frac{c}{8}, \quad \lambda\left(x^{=}\right)=m\left(x^{=}\right)
$$

and rewrite $(4.49)$ as:

$$
\left(\partial_{=} \varphi\right)=-\frac{c}{8}+e^{2 \varphi}\left(\frac{\Lambda}{4}+\frac{m\left(x^{=}\right)}{2}\right)
$$

The left-hand side of this expression looks very similar to the conformal factor $l(\varphi)$ for the black hole solution. The only difference appears to be in the extra factor $1 / 2$ and in the dependence of $m$ on the coordinate $x^{=}$. In fact, the extra numerical factor is needed for (4.44) to agree with (4.18), and the mass of the solution we are constructing indeed is not expected to be constant because of the shock wave.

The evolution of the mass can be found explicitly from the remaining equation (4.45). Using (4.48), we can rewrite the left-hand side of (4.45) as:

$$
\begin{aligned}
\frac{1}{2}\left(\partial_{=} \varphi\right) \partial_{=}\left[\ln \left(\partial_{=} \varphi\right)-\ln \left(\partial_{\mp} \varphi\right)\right] & =\frac{1}{2} \partial_{=}^{2} \varphi-\left(\partial_{=} \varphi\right)^{2}-\left(\partial_{=} \varphi\right)\left(\frac{\Lambda}{8} e^{2 \varphi}-\eta\right)= \\
& =\frac{1}{2} \partial_{=}^{2} \varphi-\left(\partial_{=} \varphi\right)^{2}-\left(\partial_{=} \varphi\right)\left(\frac{\Lambda}{8} e^{2 \varphi}+\frac{c}{8}\right)= \\
& =\frac{1}{4} e^{2 \varphi}\left(\partial_{=} m\right)
\end{aligned}
$$

Therefore, (4.43) and (4.45) imply that $m\left(x^{=}\right)$is given by the step function:

$$
m\left(x^{=}\right)=m_{0}+m_{f} \theta\left(x^{=}-x_{0}^{\overline{=}}\right)
$$




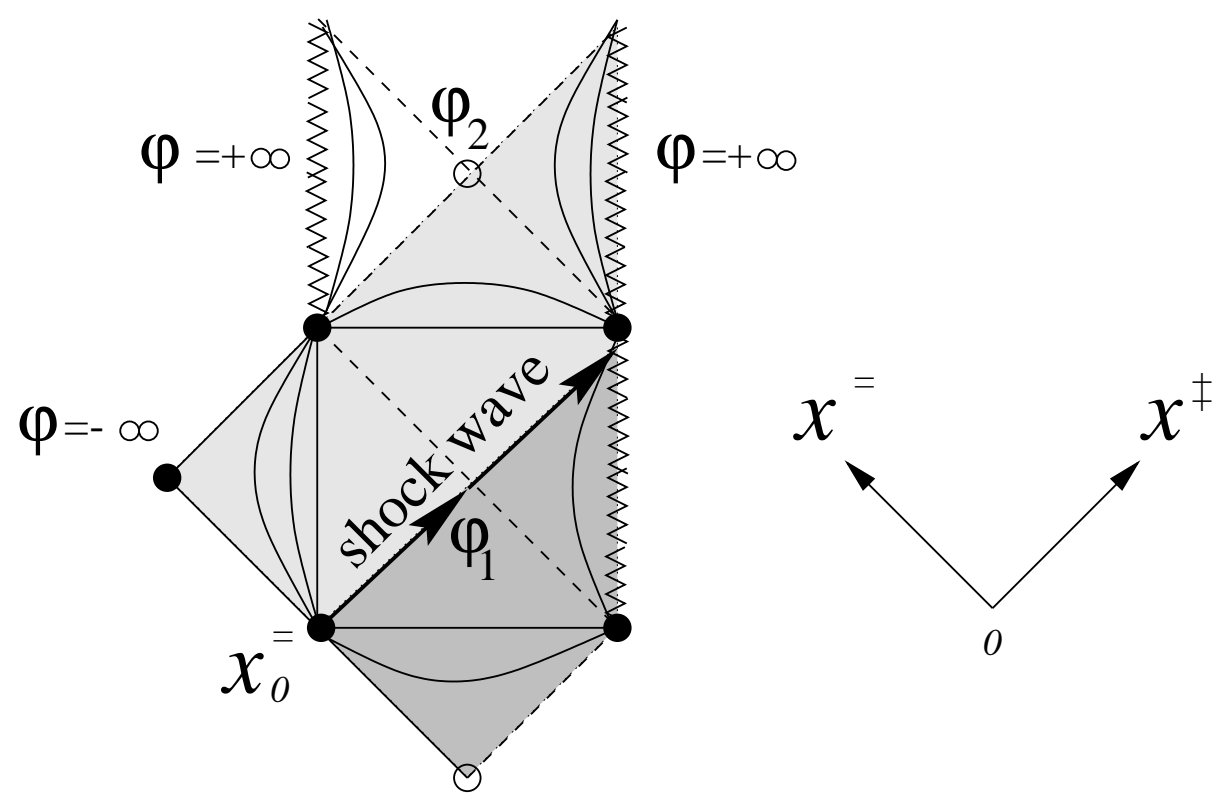

Fig. 4: Conformal diagram for the dynamical formation of the near-extremal black hole from the interaction of the shock wave with the extremal solution. The shaded regions, representing the extremal solution (before the interaction) and nonextremal solutions (after the interaction), are glued along the shock wave trajectory.

Before and after $x_{0}^{\overline{\bar{y}}}$, the solution has the same functional form (4.18):

$$
e^{2 \rho}=\frac{c}{4}-e^{2 \varphi}\left(\frac{\Lambda}{2} \varphi+m\right)
$$

The difference is that for $x^{=}<x_{0}^{\bar{x}}$ the mass of the black hole is equal to its initial value $M_{0}=\frac{2}{\sqrt{c}} m_{0}$, and after the interaction with the shock wave, $x^{=}>x_{0}^{\overline{=}}$, it is greater precisely by the energy of the shock wave:

$$
M=\frac{2}{\sqrt{c}}\left(m_{0}+m_{f}\right)
$$

In order to glue the solutions smoothly across $x^{=}=x_{0}^{\bar{z}}$, one has to compute $\varphi_{<}\left(x^{=}\right)$ before the interaction:

$$
\int^{\varphi<} \frac{d e^{-2 \varphi}}{\left(\frac{c}{4} e^{-2 \varphi}-\frac{\Lambda}{2} \varphi-m_{0}\right)}=x^{=}-x^{\ddagger}
$$

and evaluate this integral at $x^{=}=x_{0}^{\bar{z}}$. This gives the value of the dilaton field $\widetilde{\varphi}\left(x^{=}=x_{0}^{\bar{z}}\right)$. Substituting the result into another integral gives us a function $h\left(x^{\ddagger}\right)$ :

$$
h\left(x^{\ddagger}\right)=-x_{0}^{=}+\int^{\tilde{\varphi}\left(x^{\ddagger}\right)} \frac{d e^{-2 \varphi}}{\left(\frac{c}{4} e^{-2 \varphi}-\frac{\Lambda}{2} \varphi-m_{0}-m_{f}\right)}
$$


Now, we can write the solution $\varphi_{>}\left(x^{=}\right)$after the formation of the near-extremal black hole:

$$
\int^{\varphi>} \frac{d e^{-2 \varphi}}{\left(\frac{c}{4} e^{-2 \varphi}-\frac{\Lambda}{2} \varphi-m_{0}-m_{f}\right)}=x^{=}+h\left(x^{\ddagger}\right)
$$

The classical collapse of matter coupled to dilaton gravity is illustrated on Fig.4.

\subsection{Semiclassical Approximation}

Now we go to the next level of complication and consider quantum effects in the limit where the Planck constant $\hbar$ is small. We consider a generalization of the model (4.42) that involves $N$ matter fields $f_{i}$ and introduce a parameter $\kappa=N \hbar / 12$.

A consistent approximation that takes into account the Hawking radiation and its back reaction on the metric was suggested by CGHS [11]. It involves the limit of large $N$ with fixed $\kappa$. In this limit the quantum fluctuations of the dilaton and the metric are suppressed, and one can study the quantum fluctuations of the conformal matter semiclassically by adding to the action the trace anomaly term, which in the conformal gauge reads:

$$
\kappa\left(\partial_{\ddagger} \rho\right)\left(\partial_{=} \rho\right)
$$

This is nothing but the one-loop Polyakov action produced by integrating out conformal matter fields at one loop [47]. The full semi-classical action in the conformal gauge is then:

$$
\begin{aligned}
L_{e f f}=\int d^{2} x\left[e^{-2 \varphi}(\right. & \left.-2 \partial_{\ddagger} \partial_{=} \rho+4\left(\partial_{\ddagger} \varphi\right)\left(\partial_{=} \varphi\right)+\frac{c}{4} e^{2 \rho}\right)+\frac{\Lambda}{4} e^{2 \rho}- \\
& \left.-\frac{1}{2} \sum_{i=1}^{N}\left(\partial_{\ddagger} f_{i}\right)\left(\partial_{=} f_{i}\right)+\kappa\left(\partial_{\ddagger} \rho\right)\left(\partial_{=} \rho\right)\right]
\end{aligned}
$$

Since the matter fields are conformal and the one-loop anomaly term does not couple to the dilaton, the equations of motion for the dilaton and matter fields are the same as in the classical theory, $c f$. (4.6):

$$
e^{-2 \varphi}\left(-2 \partial_{\ddagger} \partial_{=} \varphi+2\left(\partial_{\ddagger} \varphi\right)\left(\partial_{=} \varphi\right)+\partial_{\ddagger} \partial_{=} \rho+\frac{c}{8} e^{2 \rho}\right)=0
$$

and

$$
\partial_{\ddagger} \partial_{=} f_{i}=0
$$

The general solution to the matter field equations looks like:

$$
f_{i}=f_{i}^{\ddagger}\left(x^{\ddagger}\right)+f_{i}^{=}\left(x^{=}\right)
$$


The other equations of motion and the constraint equations take the form:

$$
\begin{aligned}
& T_{\neq=}=e^{-2 \varphi}\left(2 \partial_{\ddagger} \partial_{=} \varphi-4\left(\partial_{\ddagger} \varphi\right)\left(\partial_{=} \varphi\right)-\frac{c}{4} e^{2 \rho}\right)-\frac{\Lambda}{4} e^{2 \rho}-\kappa\left(\partial_{\ddagger} \partial_{=} \rho\right)=0 \\
& T_{\neq \neq}=e^{-2 \varphi}\left(4\left(\partial_{\ddagger} \varphi\right)\left(\partial_{\ddagger} \rho\right)-2 \partial_{\ddagger}^{2} \varphi\right)+\frac{1}{2} \sum_{i=1}^{N}\left(\partial_{\ddagger} f_{i}\right)\left(\partial_{\ddagger} f_{i}\right)- \\
& -\kappa\left(\left(\partial_{\mp} \rho\right)\left(\partial_{\mp} \rho\right)-\partial_{\ddagger}^{2} \rho+t_{\ddagger}\left(x^{\ddagger}\right)\right)=0 \\
& T_{==}=e^{-2 \varphi}\left(4\left(\partial_{=} \varphi\right)\left(\partial_{=} \rho\right)-2 \partial_{=}^{2} \varphi\right)+\frac{1}{2} \sum_{i=1}^{N}\left(\partial_{=} f_{i}\right)\left(\partial_{=} f_{i}\right)- \\
& -\kappa\left(\left(\partial_{=} \rho\right)\left(\partial_{=} \rho\right)-\partial_{=}^{2} \rho+t_{=}\left(x^{=}\right)\right)=0
\end{aligned}
$$

where two arbitrary functions $t_{\ddagger}\left(x^{\ddagger}\right)$ and $t_{=}\left(x^{=}\right)$must be determined by boundary conditions, in particular, by the choice of incoming and outgoing states. The presence of these functions is needed in order to compensate the anomalous transformation law of the one-loop term.

In principle, one could try to study the formation and evaporation of the near-extremal black hole in this quantum system with the boundary conditions, for example, corresponding to the shock wave propagating along $x^{\ddagger}$ at $x^{=}=x_{0}^{\bar{y}}$, as in the previous subsection. The partial differential equations describing this process are (4.54) and (4.57):

$$
\partial_{\ddagger} \partial=\rho=\frac{-2\left(\partial_{\ddagger} \varphi\right)\left(\partial_{=} \varphi\right)-\frac{1}{8} e^{2 \rho}\left(c+2 \Lambda e^{2 \varphi}\right)}{\kappa e^{2 \varphi}-1}
$$

and

$$
\partial_{\ddagger} \partial_{=} \varphi=2\left(\partial_{\ddagger} \varphi\right)\left(\partial_{=} \varphi\right)+\frac{1}{8} e^{2 \rho}\left(c+\Lambda e^{2 \varphi}\right)+\frac{\kappa}{2} e^{2 \varphi} \partial_{\ddagger} \partial_{=} \rho
$$

Although hard to solve analytically, this equations can be approached using numerical methods developed for general dilaton gravity theories 48,35. We will not pursue this direction here.

Let us briefly discuss important aspects of the semi-classical dynamics that follow from the effective Lagrangian (4.53). First, we note that as $\varphi \rightarrow-\infty$ the one-loop term proportional to $\kappa$ becomes negligible (as well as the $\Lambda$-term). Therefore, in this limit the solution to the semi-classical action (4.53) should approach the classical linear dilaton vacuum. 
At the other infinity, $\varphi \rightarrow+\infty$, the semi-classical solution is asymptotic to the de Sitter space. Indeed, in this limit the $\Lambda$-term and the one-loop term are dominant, and from the equation for the conformal part of the metric (4.57) we get:

$$
-\frac{\Lambda}{4} e^{2 \rho}=\kappa \partial_{\mp} \partial_{=} \rho
$$

Substituting this into (4.4), we find the asymptotic value of the curvature as $\varphi \rightarrow+\infty$ :

$$
R \approx \frac{-2 \Lambda}{\kappa}=\frac{-24 \Lambda}{N \hbar}
$$

Since $\Lambda$ is assumed to be negative (for otherwise we had no extremal solution), locally we get a de Sitter space. It is curious to note that this de Sitter space is generated entirely by quantum effects (some other interesting quantum phenomena can be found in [49]). Therefore, it is natural to expect that its entropy, proportional to $N$, can be microscopically understood as quantum entaglement with degrees of freedom hidden behind the horizon [50]. It would be interesting to verify this by a direct calculation, analogous to [51].

Note also, that the curvature (4.62) of this semiclassical de Sitter space is inversely proportional to the number, $N$, of matter fields. This relation is reminiscent of the $\Lambda-N$ correspondence proposed by T. Banks [52], although one should remember that the model we are considering has infinite-dimensional Hilbert space (in fact, each scalar field $f_{i}$ has infinite-dimensional Hilbert space.)

The strong coupling region is described by the Liouville gravity with positive cosmological constant and conformal matter:

$$
L_{e f f}(\varphi \rightarrow+\infty) \approx \int d^{2} x\left[\frac{\Lambda}{4} e^{2 \rho}-\frac{1}{2} \sum_{i=1}^{N}\left(\partial_{\mp} f_{i}\right)\left(\partial_{=} f_{i}\right)+\kappa\left(\partial_{\ddagger} \rho\right)\left(\partial_{=} \rho\right)\right]
$$

Since this theory has massive excitations, one might expect that a shock wave propagating along $x^{\ddagger}$ will radiate part of its energy to $\mathcal{I}_{L}^{\ddagger}=\left\{x^{=} \rightarrow+\infty\right\}$, but part can be carried into the Liouville region, so one might expect some stable remnants of the black hole evaporation, unlike the CGHS case [11].

However, this part of space is separated from the weak coupling region by a curvature singularity at:

$$
e^{-2 \varphi}=\kappa
$$

This strong coupling singularity is actually present also in the CGHS solution and becomes visible at some stage of the black hole evaporation [12]. The reason is that CGHS solution has finite evaporation rate when the dilaton field at the horizon approaches the critical value (4.63). Our black hole solution would have the same fate, unless the rate of radiation goes to zero at some point. If this happens, the singularity can be hidden behind the event horizon. Below we present some arguments that this is indeed the case. 


\subsection{Evaporation of the Near-Extremal Black Hole}

Our original classical Lagrangian (4.1) is a specific example of a non-singular Lagrangians studied by Banks and O'Loughlin [34]. The authors of [34] argued that, in general, the Lagrangians like (4.1) are expected to have extremal solutions, which are nonsingular analogues of the extremal Reissner-Nordstrom solutions, and moreover that these solutions are the endpoint of the Hawking evaporation when the models are coupled to matter. Here we present some arguments that the black hole solutions (4.17) studied here, which saturate the extremality condition (4.22) are indeed the terminal points of the evaporation of non-extremal solutions produced, say, via collapse of an $f$-wave, see Fig.4. A similar problem of a charged gravitational collapse with the full nonlinear semiclassical back-reaction was studied recently in [53].

First, let us neglect the back reaction of the Hawking radiation. Then, the evaporation rate is given by the value of the energy flux across $\mathcal{I}_{L}^{\ddagger}$ at the horizon. Since the black hole solution (4.18) obeys the classical equations of motion, the first line in (4.58) is zero, and we get:

$$
\left\langle T_{\neq \ddagger}\right\rangle=-\kappa\left(\left(\partial_{\ddagger} \rho\right)\left(\partial_{\ddagger} \rho\right)-\partial_{\ddagger}^{2} \rho\right)
$$

The right-hand side of this expression is supposed to be evaluated for the classical solution (4.18). In order to do this, we use (4.15) to express the derivatives of $\rho$ in terms of $l(\varphi)$ :

$$
\begin{gathered}
\partial_{\ddagger} \rho=\frac{1}{4 l} \frac{d l}{d \varphi} \frac{d \varphi}{d x}=\frac{1}{4} \frac{d l}{d \varphi} \\
\partial_{\ddagger}^{2} \rho=\frac{1}{8} \frac{d}{d x}\left(\frac{d l}{d \varphi}\right)=\frac{1}{8} l \frac{d^{2} l}{d \varphi^{2}}
\end{gathered}
$$

Therefore, we can write (4.64) as [39]:

$$
\left\langle T_{\neq \neq}\right\rangle=-\frac{\kappa}{8}\left(2\left(l^{\prime}\right)^{2}-l^{\prime \prime} l\right)
$$

To get the evaporation rate we have to evaluate this expression at the horizon, where $l(\varphi)=0$ :

$$
\left\langle T_{\neq \neq}\right\rangle_{h}=-\frac{\kappa}{4}\left(l^{\prime}\right)^{2}=-\frac{\kappa}{4} e^{4 \varphi}\left(\Lambda \varphi+2 m+\frac{\Lambda}{2}\right)^{2}
$$

If we compare the right-hand side of this expression with the formula (4.23) for the geometric Hawking temperature, we find [39]:

$$
\left\langle T_{\neq \ddagger}\right\rangle_{h}=-\frac{\kappa}{4} T_{H}^{2}
$$


It follows that the evaporation rate is zero precisely for the extremal black hole, i.e. when both $l(\varphi)$ and $l^{\prime}(\varphi)$ vanish at the horizon. Therefore, the extremal solution (4.18) with the critical mass (4.22) is expected to be a stable remnant of the evaporation of non-extremal black hole. Quantum fields in this gravitational background will not Hawking radiate.

To provide a further evidence for this picture, we consider adiabatic evaporation of a black hole with the mass slightly above extremal:

$$
m>m_{0}=-\frac{\Lambda}{4}-\frac{\Lambda}{4} \ln \left(-\frac{c}{\Lambda}\right)
$$

For example, this black hole can be produced in the collapse of the shock wave, as we discussed above.

In the rest of this section we use the light-cone gauge, which turns out to be more convenient for this calculation. For example, the black hole metric in this gauge looks like:

$$
d s^{2}=2 d v d r+l(r) d v^{2}, \quad \varphi=r
$$

and the shock wave is described by $\epsilon^{\mu} \epsilon^{\nu} T_{\mu \nu}^{f}=2 m_{f} \delta\left(v-v_{0}\right)$, where $\epsilon=(l / 2,1)$ is a null vector. The scalar curvature in the light-cone gauge is given simply by the second derivative of the function $l$.

Following [54,35], we introduce:

$$
\Sigma=2 \partial_{v} \varphi+l \partial_{r} \varphi
$$

so that a horizon is given by $\Sigma=0$. Before the interaction with the shock wave, the space-time is described by the extremal solution (4.18) with the critical mass parameter $m_{0}$ and the apparent horizon at (4.21):

$$
\varphi_{0}=\frac{1}{2} \ln \left(-\frac{c}{\Lambda}\right)
$$

After the interaction takes place, the horizon splits into "inner" and "outer" horizons, located at $\varphi_{+}$and $\varphi_{-}$. Let us compute $\varphi_{ \pm}$in the approximation $m_{f} \ll \Lambda$. In this approximation, the values of $\varphi_{ \pm}$are supposed to be close to the original position of the horizon, $\varphi_{0}$.

Since both $l\left(\varphi_{0}\right)$ and $l^{\prime}\left(\varphi_{0}\right)$ vanish for the extremal solution, from (4.68) we have:

$$
\Sigma\left(\varphi \approx \varphi_{0}\right)=\frac{1}{2}\left(\varphi-\varphi_{0}\right)^{2} l^{\prime \prime}\left(\varphi_{0}\right)=\frac{c}{2}\left(\varphi-\varphi_{0}\right)^{2}
$$


On the other hand, the discontinuity of $\Sigma$ across the shock wave is given by [35]:

$$
\delta \Sigma=\left.\frac{2 m_{f} \exp (2 \varphi)}{\sqrt{1-\kappa \exp (2 \varphi)}}\right|_{\varphi=\varphi_{0}} \approx-2 m_{f} \frac{c}{\Lambda}
$$

The new positions of the horizon are given by $\Sigma+\delta \Sigma=0$. Therefore, substituting (4.70) into (4.69), we obtain:

$$
\varphi_{ \pm}=\varphi_{0} \pm \sqrt{-\frac{4 m_{f}}{\Lambda}}
$$

Furthermore, from (4.69) it follows that:

$$
\partial_{\varphi} \Sigma\left(\varphi_{ \pm}\right) \approx \pm\left(\varphi_{ \pm}-\varphi_{0}\right) l^{\prime \prime}\left(\varphi_{ \pm}\right)= \pm c \sqrt{-\frac{4 m_{f}}{\Lambda}}
$$

In order to find the adiabatic evolution of the $\varphi_{ \pm}$, we also need to find $\partial_{v} \Sigma\left(\varphi_{ \pm}\right)$. This can be obtained from the following linear combination of the constraint equations (4.57) $(4.59)$ :

$$
\begin{aligned}
\epsilon^{\mu} \epsilon^{\nu} T_{\mu \nu} & =\frac{\kappa}{4}\left(\frac{l}{2} \partial_{\varphi}^{2} l+\partial_{v} \partial_{\varphi} l-\frac{1}{4}\left(\partial_{\varphi} l\right)^{2}\right)= \\
& =e^{-2 \varphi}\left(\partial_{v} \Sigma+\frac{l}{2} \partial_{\varphi} \Sigma-\frac{1}{2} \Sigma^{2}-\frac{1}{2} \partial_{\varphi} l \Sigma\right)
\end{aligned}
$$

Here, we took into account that the near-extremal solution obeys classical constraint equations, and also that $t_{\varphi}$ is zero for static solutions with no net flux at infinity [35]. If we evaluate this constraint equation for $\varphi=\varphi_{ \pm}$, the leading order terms for small $m_{f}$ give:

$$
\partial_{v} \Sigma\left(\varphi_{ \pm}\right)=\frac{\kappa}{4} e^{2 \varphi} \frac{\delta l}{2} l^{\prime \prime}
$$

The discontinuity of $l(\varphi)$ across the shock wave is given by [35]:

$$
\delta l=\frac{8 m_{f}}{\kappa} \int d \varphi\left(-1+\frac{1}{\sqrt{1-\kappa \exp (2 \varphi)}}\right) \approx 2 m_{f} e^{2 \varphi}
$$

If we substitute this into (4.74) and evaluate the result at the new horizon $\varphi=\varphi_{ \pm}$, we get:

$$
\partial_{v} \Sigma\left(\varphi_{ \pm}\right)=\frac{\kappa m_{f} c^{3}}{2 \Lambda^{2}}
$$

Now we are ready to put things together, and compute the relative positions of the horizons as a function of $v$. In fact, in the adiabatic approximation we can write:

$$
\partial_{v} \varphi_{ \pm}=-\frac{\partial_{v} \Sigma}{\partial_{\varphi} \Sigma}=\frac{\kappa c^{2}}{8 \Lambda}\left(\varphi_{ \pm}-\varphi_{0}\right)
$$


The approximation we are using here holds if positions of the horizons are moving slowly enough. This implies the consistency condition $\kappa c^{2} / \Lambda^{2} \ll 1$. When this condition is satisfied, we can integrate (4.76) with the result:

$$
\varphi_{ \pm}=\varphi_{0} \pm\left(\delta \varphi_{0}\right) e^{\frac{\kappa c^{2}}{8 \Lambda}\left(v-v_{0}\right)}
$$

This means that the black hole mass goes back to the extremal value as:

$$
M(v)=M_{0}+M_{f} e^{\frac{\kappa c^{2}}{4 \Lambda}\left(v-v_{0}\right)}
$$

The Penrose diagram in the coordinates $(v, \varphi)$ for this evaporation process is almost identical to the corresponding diagram for the evaporating Reissner-Nordstrom black hole [54].

\section{Discussion}

In this paper we proposed a hybrid formalism for the manifestly spacetime supersymmetric quantization of the superstring in curved two-dimensional backgrounds with Ramond-Ramond flux, thus extending the list of existing covariant descriptions of superstrings in four [5], six [2, 3], and ten [55] space-time dimensions. Such two-dimensional backgrounds appear, for example, in Type IIA string theory on Calabi-Yau four-folds, where R-R flux is required in general for the global anomaly cancellation condition [56,57]:

$$
\frac{\chi}{24}=N+\frac{1}{2(2 \pi)^{2}} \int_{X} F^{(4)} \wedge F^{(4)}
$$

Here $\chi(X)$ is the Euler number of the Calabi-Yau four-fold $X, N$ is the number of fundamental strings filling two-dimensional space-time, and $F^{(4)}$ is the value of the background Ramond-Ramond 4-form flux. So, when $N \neq \frac{\chi}{24}$, this consistency condition requires nonzero R-R flux. One might hope to study certain aspects of these models using the covariant approach, as in section 2.7 .

Another interesting example of curved two-dimensional backgrounds discussed in this paper is a new black hole solution constructed in section 4. This application is not expected to be related to CY four-fold compactifications, since the latter leads to an effective action (4.1) with zero $c$-term, at least to the leading order in $\alpha^{\prime}$. We expect, however, that the black hole solution of (4.17) - 4.18) can be described by superstring theory on some supergroup manifold, in analogy with the $S L(2, R) / U(1)$ gauged WZW model of [10] for the $\Lambda=0$ black hole solutions. 
In principle, one can obtain this supergroup manifold (to leading order in $\alpha^{\prime}$ ) by plugging the black hole solution of (4.17) - 4.18) into the background superfields which appear in the sigma model action of (3.1) and (3.5). However, since the solution is rather complicated (e.g. it contains a non-constant dilaton), it may be simpler to try to guess the appropriate supergroup. One natural guess for the supergroup manifold is a gauged WZW model based on the supergroup

$$
G / H=O S p(1,1 \mid 2) / S O(1,1) \times S O(2) .
$$

Indeed, this coset supermanifold has the right dimension to describe an $N=(2,2)$ target superspace, viz. two bosonic and four fermionic generators. However, the WZW model based on this supergroup can be shown to be equivalent to the bosonic $S L(2, R)$ WZW model plus a set of free fermions [58]. This result is similar to the hybrid version of the sigma model action for an $A d S_{3} \times S^{3}$ background with pure NS-NS flux [3] where the $P S U(2 \mid 2) \mathrm{WZW}$ model is equivalent to the bosonic $S U(2) \times S U(1,1)$ WZW model plus a set of free fermions.

So in analogy with the $A d S_{3} \times S^{3}$ sigma model, one does not expect the gauged WZW model based on the supergroup of (5.2) to describe solutions with $\mathrm{R}-\mathrm{R}$ flux. However, in the $A d S_{3} \times S^{3}$ case, it was possible to introduce R-R flux by including additional $P S U(2 \mid 2)$ invariant terms in the action which spoil the holomorphic structure of the WZW currents but do not break the worldsheet $N=(2,2)$ superconformal invariance. So it is reasonable to expect that one can include R-R flux in the black hole solutions by introducing similar $O S p(1,1 \mid 2)$-invariant terms into the gauged WZW model based on the supergroup of (5.2). It would be very useful to find the explicit form of such terms and a preliminary search has already begun [59]. One might also hope to find a matrix model for the black hole (4.17) (4.18) in an R-R background, which in the limit $\Lambda \rightarrow 0$ would reproduce the matrix model of Kazakov, Kostov, and Kutasov [60].

\section{Acknowledgments}

We are grateful to C. G. Callan, O. Chandía, A.T. Filippov, G.T. Horowitz, J. Maldacena, D. Nedel, R. Plesser, H. Ooguri, V. Rivelles, J.H. Schwarz, N. Seiberg, S. Shenker, A. Strominger, C. Vafa, and E. Witten for useful discussions and comments. The work of N.B. is supported in part by CNPq grant 300256/94-9, Pronex 66.2002/1998-9, and FAPESP grant 99/12763-0. This research was partially conducted during the period N.B. served as a Clay Mathematics Institute Prize Fellow and S.G. served as a Clay Mathematics Institute Long-Term Prize Fellow. The work of S.G. is also supported in part by 
the Caltech Discovery Fund, NSF grant No. PHY99-07949, grant RFBR No. 01-02-17488, and the Russian President's grant No. 00-15-99296. The work of B.C.V. is supported by FAPESP grant No 00/02230-3. N.B. and S.G. are grateful to the California Institute of Technology, where part of this work was done, for hospitality. 


\section{References}

[1] N. Berkovits and C. Vafa, "On the Uniqueness of String Theory", Mod. Phys. Lett. A9 (1994) 653, hep-th/9310129.

[2] N. Berkovits and C. Vafa, " $N=4$ Topological Strings" , Nucl. Phys. B433 (1995) 123.

[3] N. Berkovits, C. Vafa and E. Witten, "Conformal Field Theory of AdS Background with Ramond-Ramond Flux", JHEP 9903 (1999) 018.

[4] N. Berkovits, "Quantization of the Type II Superstring in a Curved Six-Dimensional Background", Nucl. Phys. B565 (2000) 333, hep-th/9908041.

[5] N. Berkovits, "Covariant Quantization of the Green-Schwarz Superstring in a CalabiYau Background", Nucl. Phys. B431, 258 (1994).

[6] N. Berkovits and W. Siegel, "Superspace Effective Actions for 4D Compactifications of Heterotic and Type II Superstrings", Nucl. Phys. B462 (1996) 213, hep-th/9510106.

[7] C. Vafa, "Superstrings and Topological Strings at Large N," hep-th/0008142.

[8] S. Elitzur, A. Forge and E. Rabinovici, "Some global aspects of string compactifications," Nucl. Phys. B359, 581 (1991).

[9] G. Mandal, A. M. Sengupta and S. R. Wadia, "Classical solutions of two-dimensional string theory," Mod. Phys. Lett. A6, 1685 (1991).

[10] E. Witten, "On String Theory and Black Holes," Phys.Rev. D44 (1991) 314.

[11] C. Callan Jr., S.B. Giddings, J.A. Harvey and A. Strominger, "Evanescent Black Holes", Phys. Rev. D45 (1992) 1005, hep-th/9111056.

[12] S. W. Hawking, "Evaporation of Two Dimensional Black Holes," Phys.Rev.Lett. 69 (1992) 406.

[13] M.D. McGuigan, C.R. Nappi, S.A. Yost, "Charged Black Holes in Two-Dimensional String Theory," Nucl. Phys. B375 (1992) 421.

[14] D. Friedan, E. Martinec and S. Shenker, "Conformal Invariance, Supersymmetry and String Theory", Nucl. Phys. B271 (1986) 93.

[15] N. Berkovits, "Off-Shell Supersymmetry versus Hermiticity in the Superstring", Phys. Rev. Letters 77 (1996) 2891, hep-th/9604121.

[16] S. Gukov, "Solitons, Superpotentials and Calibrations," Nucl.Phys. B574 (2000) 169.

[17] W. Lerche, C. Vafa and N.P. Warner, "Chiral Rings in N=2 Superconformal Theories", Nucl.Phys. B324 (1989) 427.

[18] L. Dixon, Lectures at the 1987 ICTP summer Workshop in High Energy Physics and Cosmology.

[19] S.-T. Yau, editor, Essays on Mirror Manifolds, International Press, 1992; B. Greene and S.-T. Yau, editors, Mirror Symmetry. II, International Press, 1997.

[20] B.R. Greene, D.R. Morrison and M.R. Plesser, "Mirror Manifolds in Higher Dimension", Commun.Math.Phys. 173 (1995) 559. 
[21] H. Ooguri, Y. Oz and Z. Yin, "D-Branes on Calabi-Yau Spaces and Their Mirrors", Nucl.Phys. B477 (1996) 407.

[22] E. Witten, "String Theory Dynamics In Various Dimensions," Nucl.Phys. B443 (1995) 85.

[23] E. Bergshoeff, R. Kallosh, T. Ortin, D. Roest and A. Van Proeyen, "New Formulations of $\mathrm{D}=10$ Supersymmetry and D8-O8 Domain Walls," hep-th/0103233.

[24] S.J. Gates, Jr., S. Gukov and E. Witten "Two Two-Dimensional Supergravity Theories from Calabi-Yau Four-Folds", Nucl.Phys. B584 (2000) 109.

[25] S. Gukov, C. Vafa and E. Witten, "CFT's From Calabi-Yau Four-folds", hepth/9906070.

[26] M. Haack and J. Louis, "M-theory compactified on Calabi-Yau fourfolds with background flux," hep-th/0103068.

[27] L.J. Dixon, V. Kaplunovsky and J. Louis, "On Effective Field Theories Describing $(2,2)$ Vacua of the Heterotic String", Nucl.Phys. B329 (1990) 27.

[28] B. de Wit, P. G. Lauwers and A. Van Proeyen, "Lagrangians of N=2 Supergravity Matter Systems", Nucl.Phys. B255 (1985) 569.

[29] W. Lerche, "Fayet-Iliopoulos Potentials from Four-Folds", JHEP 9711 (1997) 004.

[30] J. de Boer and K. Skenderis, "Covariant Computation of the Low Energy Effective Action of the Heterotic Superstring", Nucl. Phys. B481 (1996) 129, hep-th/9608078.

[31] M.T. Grisaru and M.E. Wehlau, "Superspace Measures, Invariant Actions, and Component Projection Formulae for (2,2) Supergravity", Nucl.Phys. B457 (1995) 219.

[32] S.J. Gates, Jr., M.T. Grisaru and M.E. Wehlau, "A Study of General 2D, N=2 Matter Coupled to Supergravity in Superspace", Nucl.Phys. B460 (1996) 579.

[33] S. Mignemi, "Black hole solutions in generalized two-dimensional dilation gravity theories," Phys. Rev. D50 (1994) 4733.

[34] T. Banks and M. O'Loughlin, "Nonsingular Lagrangians for Two Dimensional Black Holes," Phys.Rev. D48 (1993) 698.

[35] D.A. Lowe and M. O'Loughlin, "Nonsingular Black Hole Evaporation and "Stable" Remnants," Phys.Rev. D48 (1993) 3735.

[36] Y. Park, A. Strominger, "Supersymmetry and Positive Energy in Classical and Quantum Two-Dimensional Dilaton Gravity," Phys.Rev. D47 (1993) 1569.

[37] M.O. Katanaev, "Effective action for scalar fields in two-dimensional gravity," grqc/0101033.

[38] J. Russo, A.A. Tseytlin, "Scalar-Tensor Quantum Gravity in Two Dimensions," Nucl.Phys. B382 (1992) 259.

[39] M. O. Katanaev, W. Kummer and H. Liebl, "On the completeness of the black hole singularity in 2D dilaton theories," Nucl.Phys. B486 (1997) 353.

[40] M.O. Katanaev, "Global Solutions in Gravity. Lorentzian signature," Proc.Steklov Inst.Math. 228 (2000) 158. 
[41] J. Maldacena, J. Michelson and A. Strominger, "Anti-de Sitter Fragmentation," JHEP 9902 (1999) 011.

[42] R.B. Mann, "Conservation Laws and 2D Black Holes in Dilaton Gravity," Phys.Rev. D47 (1993) 4438.

[43] H. Liebl, D.V. Vassilevich and S. Alexandrov, "Hawking radiation and masses in generalized dilaton theories," Class.Quant.Grav. 14 (1997) 889.

[44] S.N. Solodukhin, "Two-dimensional Quantum-Corrected Eternal Black Hole," Phys. Rev. D53 (1996) 824.

[45] A.T. Filippov, "Exact Solutions of $(1+1)$-Dimensional Dilaton Gravity Coupled to Matter," Mod.Phys.Lett. A11 (1996) 1691; "Integrable 1+1 dimensional gravity models," Int.J.Mod.Phys. A12 (1997) 13.

[46] O.B. Zaslavskii, "Exactly solvable models of two-dimensional dilaton gravity and quantum eternal black holes," Phys. Rev. D59 (1999) 084013; "Semi-infinite throats at finite temperature and static solutions in exactly solvable models of $2 \mathrm{~d}$ dilaton gravity," Phys.Lett. B459 (1999) 105.

[47] A.M. Polyakov, Phys.Lett. bf 103B (1981) 207.

[48] D.A. Lowe, "Semiclassical Approach to Black Hole Evaporation," Phys.Rev. D47 (1993) 2446.

[49] S. Nojiri, S.D. Odintsov, "Quantum dilatonic gravity in d $=2,4$ and 5 dimensions," Int.J.Mod.Phys. A16 (2001) 1015.

[50] L. Susskind and J. Uglum, "Black Hole Entropy in Canonical Quantum Gravity and Superstring Theory," Phys.Rev. D50 (1994) 2700; T.M. Fiola, J. Preskill, A. Strominger and S.P. Trivedi, "Black hole thermodynamics and information loss in two dimensions," Phys.Rev. D50 (1994) 3987; T. Jacobson, "Black Hole Entropy and Induced Gravity," gr-qc/9404039.

[51] S. Hawking, J. Maldacena and A. Strominger, "DeSitter entropy, quantum entanglement and ADS/CFT," JHEP 0105 (2001) 001.

[52] T. Banks, "Cosmological Breaking of Supersymmetry?," hep-th/0007146.

[53] E. Sorkin, T. Piran, "Formation and Evaporation of Charged Black Holes," Phys.Rev. D63 (2001) 124024.

[54] A. Strominger and S.P. Trivedi, "Information Consumption by Reissner-Nordstrom Black Holes," Phys.Rev. D48 (1993) 5778.

[55] N. Berkovits, "Super-Poincare Covariant Quantization of the Superstring", JHEP 04 (2000) 018, hep-th/0001035.

[56] S. Sethi, C. Vafa and E. Witten, "Constraints on Low-Dimensional String Compactifications", Nucl. Phys. B480 (1996) 213.

[57] E. Witten, "On Flux Quantization In M-Theory And The Effective Action", J. Geom. Phys. 22 (1997) 1. 
[58] I. Bars, "Free Fields and New Cosets of Current Algebras", Phys. Lett. B255 (1991) 353.

[59] N. Berkovits and S. Gukov, work in progress.

[60] V. Kazakov, I. Kostov and D. Kutasov, "A Matrix Model for the Two Dimensional Black Hole," hep-th/0101011. 